\title{
FROM KLEIN TO PAINLEVÉ VIA FOURIER, LAPLACE AND JIMBO
}

\author{
PHILIP BOALCH
}

\begin{abstract}
We will describe a method for constructing explicit algebraic solutions to the sixth Painlevé equation, generalising that of Dubrovin-Mazzocco. There are basically two steps: First we explain how to construct finite braid group orbits of triples of elements of $\mathrm{SL}_{2}(\mathbb{C})$ out of triples of generators of three-dimensional complex reflection groups. (This involves the Fourier-Laplace transform for certain irregular connections.) Then we adapt a result of Jimbo to produce the Painlevé VI solutions. (In particular this solves a Riemann-Hilbert problem explicitly.)

Each step will be illustrated using the complex reflection group associated to Klein's simple group of order 168. This leads to a new algebraic solution with seven branches. We will also prove that, unlike the algebraic solutions of Dubrovin-Mazzocco and Hitchin, this solution is not equivalent to any solution coming from a finite subgroup of $\mathrm{SL}_{2}(\mathbb{C})$.

The results of this paper also yield a simple proof of a recent theorem of InabaIwasaki-Saito on the action of Okamoto's affine $D_{4}$ symmetry group as well as the correct connection formulae for generic Painlevé VI equations.
\end{abstract}

\section{INTRODUCTION}

Klein's quartic curve

$$
X^{3} Y+Y^{3} Z+Z^{3} X=0 \quad \subset \mathbb{P}^{2}(\mathbb{C})
$$

is of genus three and has the maximum possible number $84(g-1)=168$ of holomorphic automorphisms. Klein found these automorphisms explicitly (in terms of $3 \times 3$ matrices). They constitute Klein's simple group $K \subset \mathrm{PGL}_{3}(\mathbb{C})$ which is isomorphic to $\mathrm{PSL}_{2}(7)$.

Lifting to $\mathrm{GL}_{3}(\mathbb{C})$ there is a two-fold covering group $\widehat{K} \subset \mathrm{GL}_{3}(\mathbb{C})$ of order 336 which is a complex reflection group - there are complex reflections

$$
r_{1}, r_{2}, r_{3} \in \mathrm{GL}_{3}(\mathbb{C})
$$

which generate $\widehat{K}$. (Recall a pseudo-reflection is an automorphism of the form "one plus rank one", a complex reflection is a pseudo-reflection of finite order and a complex reflection group is a finite group generated by complex reflections. Here, each generator $r_{i}$ has order two - as for real reflections.)

Using the general tools to be described in this paper we will construct, starting from the Klein complex reflection group $\widehat{K}$, another algebraic curve with affine equation

$$
F(t, y)=0
$$

given by a polynomial $F$ with integer coefficients. This curve will be a seven-fold cover of the $t$-line branched only at $0,1, \infty$ and such that the function $y(t)$, defined implicitly by (21), solves the Painlevé VI differential equation.

One upshot of this will be to construct an explicit rank three Fuchsian system of linear differential equations with four singularities (at $0, t, 1, \infty$, for some $t$ ) on $\mathbb{P}^{1}$, and with 
monodromy group equal to $\widehat{K}$ in its natural representation (so the monodromy around each of the finite singularities $0, t, 1$ is a generating reflection).

In general the construction of linear differential equations with finite monodromy group is reasonably straightforward provided one works with rigid representations of the monodromy groups. In our situation the representation is minimally non-rigid; it lives in a complex two-dimensional moduli space, and this is the basic reason the (second order) Painlevé VI equation arises.

Apart from the many physical applications, from a mathematical perspective our main interest in the Painlevé VI equation is that it is the explicit form of the simplest isomonodromy (=non-abelian Gauss-Manin) connection. In brief, the isomonodromy connections arise by replacing the closed differential forms and periods appearing in the usual (abelian) Gauss-Manin picture, by flat connections and monodromy representations, respectively.

Indeed one may view the Painlevé VI equation as a natural nonlinear analogue of the Gauss hypergeometric equation. From this point of view the thrust of this paper is towards finding the analogue of Schwartz's famous list of hypergeometric equations with algebraic solutions.

Before carefully describing the contents of this paper we will briefly recall exactly how the sixth Painlevé equation arises.

Consider a Fuchsian system of differential equations (with four singularities) of the form

$$
\frac{d \Phi}{d z}=A(z) \Phi ; \quad A(z)=\sum_{i=1}^{3} \frac{A_{i}}{z-a_{i}}
$$

where the $A_{i}$ 's are $2 \times 2$ traceless matrices. We wish to deform (3) isomonodromically-i.e. when the pole positions $\left(a_{1}, a_{2}, a_{3}\right)$ are moved in $\mathbb{C}^{3} \backslash$ diagonals we wish to vary the coefficients $A_{i}$ such that the conjugacy class of the corresponding monodromy representation is preserved. Such isomonodromic deformations are governed by Schlesinger's equations:

$$
\frac{\partial A_{i}}{\partial a_{j}}=\frac{\left[A_{i}, A_{j}\right]}{a_{i}-a_{j}} \quad \text { if } i \neq j \text {, and } \quad \frac{\partial A_{i}}{\partial a_{i}}=-\sum_{j \neq i} \frac{\left[A_{i}, A_{j}\right]}{a_{i}-a_{j}} .
$$

Let us view these more geometrically as a nonlinear connection on a fibre-bundle. First observe that Schlesinger's equations preserve the adjoint orbit $O_{i}$ containing each $A_{i}$ and are invariant under overall conjugation of $\left(A_{1}, A_{2}, A_{3}, A_{4}\right)$, where $A_{4}=-A_{1}-A_{2}-A_{3}$ is the residue of (B) at infinity. Thus one sees that Schlesinger's equations amount to a flat connection, the isomonodromy connection, on the trivial fibre bundle

$$
\mathcal{M}^{*}:=\left(O_{1} \times O_{2} \times O_{3} \times O_{4}\right) / / G \times B \longrightarrow B
$$

over $B:=\mathbb{C}^{3} \backslash$ diagonals, where the fibre $\left(O_{1} \times \cdots \times O_{4}\right) / / G$ is the quotient of

$$
\left\{\left(A_{1}, A_{2}, A_{3}, A_{4}\right) \in O_{1} \times O_{2} \times O_{3} \times O_{4} \mid \sum A_{i}=0\right\}
$$

by overall conjugation by $G=\mathrm{SL}_{2}(\mathbb{C})$. (Generically this fibre is two dimensional and has a natural complex symplectic structure.)

Now for each point $\left(a_{1}, a_{2}, a_{3}\right)$ of the base $B$ one can also consider the set

$$
\operatorname{Hom}_{\mathcal{C}}\left(\pi_{1}\left(\mathbb{C} \backslash\left\{a_{i}\right\}\right), G\right) / G
$$


of conjugacy classes of representations of the fundamental group of the four-punctured sphere, where the representations are restricted to take the simple loop around $a_{i}$ into the conjugacy class $\mathcal{C}_{i}:=\exp \left(2 \pi \sqrt{-1} O_{i}\right) \subset G\left(i=1, \ldots, 4, a_{4}=\infty\right)$. These spaces of representations are also generically two dimensional (and complex symplectic) and fit together into a fibre bundle

$$
M \longrightarrow B \text {. }
$$

Moreover this bundle $M$ has a complete flat connection defined locally by identifying representations taking the same values on a fixed set of fundamental group generators. The isomonodromy connection is the pullback of this complete connection along the natural bundle map

$$
\nu: \mathcal{M}^{*} \longrightarrow M
$$

defined by taking the systems (3) to their monodromy representations (cf. [18, 4]).

To obtain Painlevé VI one chooses specific coordinates $x, y$ on the fibres of $\mathcal{M}^{*}$ and then, upon restricting the pole positions to $\left(a_{1}, a_{2}, a_{3}\right)=(0, t, 1)$, the isomonodromy connection amounts to two first order coupled nonlinear equations for $x(t), y(t)$. Eliminating $x$ yields (cf. e.g. [24]) the sixth Painlevé equation ${ }^{1}$ (PVI):

$$
\begin{aligned}
\frac{d^{2} y}{d t^{2}}= & \frac{1}{2}\left(\frac{1}{y}+\frac{1}{y-1}+\frac{1}{y-t}\right)\left(\frac{d y}{d t}\right)^{2}-\left(\frac{1}{t}+\frac{1}{t-1}+\frac{1}{y-t}\right) \frac{d y}{d t} \\
& +\frac{y(y-1)(y-t)}{t^{2}(t-1)^{2}}\left(\alpha+\beta \frac{t}{y^{2}}+\gamma \frac{(t-1)}{(y-1)^{2}}+\delta \frac{t(t-1)}{(y-t)^{2}}\right) .
\end{aligned}
$$

The four parameters $\alpha, \beta, \gamma, \delta \in \mathbb{C}$ here are directly related to the choice of the adjoint orbits $O_{i}$. From another viewpoint, we will see the monodromy spaces (6) are affine cubic surfaces and Iwasaki 22] has recently pointed out that the four parameters correspond to the moduli of such surfaces, appearing in the Cayley normal form.

The sixth Painlevé equation has critical singularities at $0,1, \infty$ and is remarkable in that any of its solutions have wonderful analytic continuation properties: any locallydefined solution $y(t)$ may be analytically continued to a meromorphic function on the universal cover of the three-punctured sphere $\mathbb{P}^{1} \backslash\{0,1, \infty\}$. (This is the so-called Painlevé property.)

From the geometric viewpoint, the monodromy of PVI (i.e. the analytic continuation of solutions around $\left.\mathbb{P}^{1} \backslash\{0,1, \infty\}\right)$ corresponds to the monodromy of the nonlinear connection on $\mathcal{M}^{*}$. In turn this connection is the pullback of the complete connection on the bundle $M$. Being complete, the monodromy of the connection on $M$ amounts to an action of the fundamental group of the base $B$ (the pure braid group $\mathcal{P}_{3}$ ) on the standard fibre (6). This is the standard braid group action on the monodromy data, which we thus see gives the monodromy of solutions to PVI. ${ }^{2}$

Our main concern in this paper is to construct algebraic solutions to PVI. One knows that, for generic values of the four parameters, any solution of PVI is a 'new transcendental function' on the universal cover of the three-punctured sphere. However, for special

\footnotetext{
${ }^{1}$ The general PVI equation was first written down by R. Fuchs (son of L. Fuchs) and it was added to the list of Painlevé equations by Painlevé's student B. Gambier.

${ }^{2}$ Restricting to $\mathbb{P}^{1} \backslash\{0,1, \infty\} \stackrel{\iota}{\hookrightarrow} B$, where $\iota(t)=(0, t, 1)$, amounts to restricting to the action of the free subgroup $\mathcal{F}_{2}:=\pi_{1}\left(\mathbb{P}^{1} \backslash\{0,1, \infty\}\right) \stackrel{\iota_{*}^{*}}{\hookrightarrow} \mathcal{P}_{3}$ of the braid group. This $\mathcal{F}_{2}$ action is equivalent to the $\mathcal{P}_{3}$ action since the centre $Z \cong \mathbb{Z}$ of $\mathcal{P}_{3}$ acts trivially and $\iota_{*}$ induces an isomorphism $\mathcal{F}_{2} \cong \mathcal{P}_{3} / Z$.
} 
values of the parameters it is possible that there are solutions expressible in terms of standard transcendental functions, or even solutions which are algebraic - i.e. are defined by polynomial equations. For example there are the algebraic solutions of Hitchin [19, 20, Dubrovin [11] and Dubrovin-Mazzocco [13] related to the dihedral, tetrahedral, octahedral and icosahedral groups.

The problem of constructing algebraic solutions may be broken into two steps. First, the algebraic solutions will have a finite number of branches and so one may start by looking for finite orbits of the $\mathcal{P}_{3}$ action on the space of monodromy data (6).

Clearly if we start with a linear system (3) whose monodromy is a finite subgroup of $\mathrm{SL}_{2}(\mathbb{C})$, then the corresponding braid group orbit will be finite. The solutions of Hitchin, Dubrovin and Mazzocco mentioned above are equivalent to solutions arising in this way.

The basic idea underlying the present paper is that PVI also arises as the equation for isomonodromic deformations of certain rank three Fuchsian systems. Namely we replace $A_{1}, A_{2}, A_{3}$ in (3) by $3 \times 3$ matrices $B_{1}, B_{2}, B_{3}$ each of rank one. Then the corresponding moduli spaces are still of dimension two, and one finds again that PVI governs the isomonodromic deformations (and that any PVI equation arises in this way). Note that the rank one condition implies the corresponding monodromy group will be generated by a triple of pseudo-reflections in $\mathrm{GL}_{3}(\mathbb{C})$.

Rather than work throughout with this equivalent $3 \times 3$ representation of PVI, we will pass between the two pictures in order to use existing machinery developed in the $2 \times 2$ framework (in particular the work of Jimbo [23]).

Our starting point will be to describe a method of constructing finite braid group orbits of triples of elements of $\mathrm{SL}_{2}(\mathbb{C})$ starting from any triple of complex reflections generating a complex reflection group in $\mathrm{GL}_{3}(\mathbb{C})$. In general this will yield more exotic finite braid group orbits than those from finite subgroups of $\mathrm{SL}_{2}(\mathbb{C})$. The key idea behind this construction is to use the Fourier-Laplace transformation to convert the rank three Fuchsian system into a rank three system with an irregular singularity, then to apply a simple scalar shift and transform back, so that the resulting Fuchsian system is reducible, and we take the irreducible rank two quotient or subsystem. Of crucial importance here is Balser-Jurkat-Lutz's computation [1] of the action of the Fourier-Laplace transformation on monodromy data, relating the monodromy data of the Fuchsian system to the Stokes data $u_{ \pm}$of the irregular system. This correspondence may be described by the explicit formula

$$
r_{3} r_{2} r_{1}=u_{-}^{-1} t^{2} u_{+}
$$

(dating back at least to Killing) for the Birkhoff factorisation of the product of generating reflections, and enables us to compute the action of the scalar shift on the reflections.

Lots of finite braid group orbits of $\mathrm{SL}_{2}(\mathbb{C})$ triples are obtained in this way: We recall that Shephard-Todd [31] have classified all the complex reflection groups and showed that in three-dimensions, apart from the real reflection groups, there are four irreducible complex reflection groups generated by triples of reflections, of orders 336, 648, 1296 and 2160 respectively, as well as two infinite families $G(m, p, 3), m \geq 3, p=1, m$ of groups of orders $6 m^{3} / p$. For $m=2$ and $p=1,2$ these would be the symmetry groups of the octahedron and tetrahedron respectively. (In general, for other $p$ dividing $m, G(m, p, 3)$ is not generated by a triple of reflections.) The main example we will focus on, the Klein group, is thus the smallest non-real exceptional complex reflection group. This leads to 
a $\mathcal{P}_{3}$ orbit of size seven which we will prove is not isomorphic to any orbit coming from a finite subgroup of $\mathrm{SL}_{2}(\mathbb{C})$.

The second step in the construction of algebraic solutions is to pass from the finite braid group orbit to the explicit solution. For this we adapt (and correct) a result of Jimbo [23] giving an explicit formula for the leading term in the asymptotic expansion at zero of the solution $y(t)$ on each branch. By using the PVI equation this is sufficient to determine the solution curve precisely:

$$
\begin{gathered}
F(t, y)= \\
\left(162 t^{3}-243 t^{2}-243 t+162\right) y^{7}+\left(-567 t^{3}+2268 t^{2}-567 t\right) y^{6}+ \\
\left(-1701 t^{3}-1701 t^{2}\right) y^{5}+\left(1407 t^{4}+2856 t^{3}+1407 t^{2}\right) y^{4}+ \\
\left(14 t^{5}-2849 t^{4}-2849 t^{3}+14 t^{2}\right) y^{3}+\left(-21 t^{5}+3444 t^{4}-21 t^{3}\right) y^{2}+ \\
\left(-567 t^{5}-567 t^{4}\right) y+\left(125 t^{6}-88 t^{5}+125 t^{4}\right)
\end{gathered}
$$

which admits the rational parameterisation:

$$
y=-\frac{\left(5 s^{2}-8 s+5\right)\left(7 s^{2}-7 s+4\right)}{s(s-2)(s+1)(2 s-1)\left(4 s^{2}-7 s+7\right)}, \quad t=\frac{\left(7 s^{2}-7 s+4\right)^{2}}{s^{3}\left(4 s^{2}-7 s+7\right)^{2}} .
$$

Using this parameterisation it is easy to carry out the ultimate test and substitute back into the Painlevé VI equation, with $(\alpha, \beta, \gamma, \delta)=(9,-4,4,45) / 98$, finding that we do indeed have a solution.

Note that we have not considered the further problem of writing down the affine Weyl group orbit of (77), or of finding the simplest representative.

The general strategy of this paper is the same as the paper [13] of Dubrovin-Mazzocco. Indeed part of our motivation was to extend their work to (a dense open subset of) the full four parameter family of PVI equations. Recall that 13 dealt with the real (orthogonal) three-dimensional reflection groups and for this it was sufficient to only consider a one-parameter family of PVI equations (corresponding to fixing each of $A_{1}, A_{2}, A_{3}$ to be nilpotent, so the remaining parameter is the choice of orbit of $A_{4}$ ).

In relation to 13 the key results of the present paper are firstly to see how to extend their method of passing from generating triples of orthogonal reflections to finite $\mathcal{P}_{3}$ orbits of (unipotent) $\mathrm{SL}_{2}(\mathbb{C})$ triples. (Reading the earlier papers [11, 12] of Dubrovin was helpful to fully understand this aspect of [13.)

Secondly we were able to fix Jimbo's asymptotic formula. (Dubrovin-Mazzocco did not use Jimbo's asymptotic result, but adapted Jimbo's argument to prove a version of it for their nilpotent situation.) The key point here was to find a sign error hidden in the depths of Jimbo's asymptotic formula - perhaps we should emphasize that without the correction the construction of this paper will not work at all. (Namely at some point we need to obtain precise rational numbers out of the transcendental formulae.) This sign is also important because it is needed to obtain the correct connection formulae for solutions of the Painlevé VI equation - by symmetry analogous asymptotic formulae may be obtained at one and at infinity, thereby giving the connection formulae.

The two main tools of this paper (construction of finite $\mathcal{P}_{3}$ orbits of $\mathrm{SL}_{2}(\mathbb{C})$ triples, and Jimbo's formula) are independent and will have separate applications. For example one may take any triple of elements of a finite subgroup of $\mathrm{SL}_{2}(\mathbb{C})$ and try to apply Jimbo's formula to find solutions to PVI. (E.g. in [2] we have classified the inequivalent $\mathcal{P}_{3}$ orbits 
of generators of the binary icosahedral group and, as a further test of Jimbo's formula, constructed a new algebraic solution to PVI with 12 branches - this is the largest genus zero icosahedral solution and is interesting since its parameters lie on none of the reflecting hyperplanes of Okamoto's affine $F_{4}$ action.)

The layout of this paper is as follows. In section 2 we explain in a direct algebraic fashion how to obtain finite braid group orbits of (conjugacy classes of) triples of elements of $\mathrm{SL}_{2}(\mathbb{C})$ from triples of generators of three-dimensional complex reflection groups. Section 3 (which could be skipped on a first reading) then explains how the formulae of section 2 were found. This is somewhat more technical, involving the action of the FourierLaplace transform on monodromy data, but is necessary to understand the origin of the procedure of section 2. We also mention in passing (Remark 22) the relation with the $\mathrm{GL}_{n}(\mathbb{C})$ quantum Weyl group actions. Next, in section 4, we give Jimbo's formula for the leading term in the asymptotic expansion at zero of the PVI solution $y(t)$ on the branch specified by a given $\mathrm{SL}_{2}(\mathbb{C})$ triple. This is applied in section 5 to find the Klein solution explicitly. Section 6 then proves that the Klein solution is not equivalent (under Okamoto's affine $F_{4}$ action) to any solution coming from a finite subgroup of $\mathrm{SL}_{2}(\mathbb{C})$. Then in section [7 we explain how to reconstruct, from such a PVI solution, an explicit rank three Fuchsian system with monodromy group generated by the triple of complex reflections we started with in section 2. Finally in section 8 we describe a direct path from the $3 \times 3$ Fuchsian isomonodromic deformations to PVI and deduce a recent theorem of Inaba-Iwasaki-Saito [21].

It should be mentioned that, in the short paper [6], we previously showed by a different method that the equations for isomonodromic deformations of the $3 \times 3$ Fuchsian systems mentioned above are equivalent to PVI - this was written before Jimbo's formula was fixed and also does not give the relation between the rank two and three monodromy data.

Acknowledgments. The Klein solution was found whilst the author was a J. F. Ritt assistant professor of Mathematics at Columbia University, New York, and was announced at the April 2003 AMS meeting at the Courant Institute. Various other parts were done whilst the author was a member of DPMMS (Cambridge), The Mathematical Institute (Oxford), SISSA (Trieste) and IRMA (Strasbourg, supported by the EDGE Research Training Network HPRNCT-2000-00101). The author is grateful to Nigel Hitchin, Boris Dubrovin and Marta Mazzocco for useful conversations, and to Anton Alekseev for inviting him to visit the University of Geneva Mathematics Department and the Erwin Schrödinger Institute (Vienna) during the summer of 2003, where this paper was written (supported by the Swiss NSF and the ESI respectively).

\section{BRAID GROUP ORBITS}

In this section we will explain how to obtain some interesting finite braid group orbits of triples of elements of $\mathrm{SL}_{2}(\mathbb{C})$ from triples of generators of three-dimensional complex reflection groups.

The motivation is simply the fact that branches of a solution to PVI are parameterised by pure braid group orbits of conjugacy classes of triples of elements of $\mathrm{SL}_{2}(\mathbb{C})$. Clearly any algebraic solution of PVI has a finite number of branches and so the first step towards finding an algebraic solution is to find a finite braid group orbit, which is what we will do here. 


\section{$2 \times 2$ case.}

Let $G=\mathrm{SL}_{2}(\mathbb{C})$ and consider the standard action of the three-string braid group $\mathcal{B}_{3}$ on $G^{3}$ generated by

$$
\begin{aligned}
& \beta_{1}\left(M_{3}, M_{2}, M_{1}\right)=\left(M_{2}, M_{2}^{-1} M_{3} M_{2}, M_{1}\right) \\
& \beta_{2}\left(M_{3}, M_{2}, M_{1}\right)=\left(M_{3}, M_{1}, M_{1}^{-1} M_{2} M_{1}\right)
\end{aligned}
$$

where $M_{i} \in G$. We are interested in the induced action of $\mathcal{B}_{3}$ on the set of conjugacy classes of such triples.

First we recall some basic facts (cf. e.g. [27]). To begin with note that the seven functions

$$
\begin{gathered}
m_{1}:=\operatorname{Tr}\left(M_{1}\right), \quad m_{2}:=\operatorname{Tr}\left(M_{2}\right), \quad m_{3}:=\operatorname{Tr}\left(M_{3}\right), \\
m_{12}:=\operatorname{Tr}\left(M_{1} M_{2}\right), \quad m_{23}:=\operatorname{Tr}\left(M_{2} M_{3}\right), \quad m_{13}:=\operatorname{Tr}\left(M_{1} M_{3}\right) \\
m_{321}:=\operatorname{Tr}\left(M_{3} M_{2} M_{1}\right) .
\end{gathered}
$$

on $G^{3}$ are invariant under the diagonal conjugation action of $G$ and in fact generate the ring of invariant polynomials. Indeed the ring of invariants is isomorphic to the quotient of $\mathbb{C}\left[m_{1}, m_{2}, m_{3}, m_{12}, m_{23}, m_{13}, m_{321}\right]$ by the (ideal generated by the) so-called Fricke relation:

$$
m_{321}^{2}-P m_{321}+Q=4
$$

where $P, Q$ are the following polynomials in the first six variables:

$$
P=m_{1} m_{23}+m_{2} m_{13}+m_{3} m_{12}-m_{1} m_{2} m_{3}
$$

$Q=m_{1}^{2}+m_{2}^{2}+m_{3}^{2}+m_{12}^{2}+m_{23}^{2}+m_{13}^{2}+m_{12} m_{23} m_{13}-m_{1} m_{2} m_{12}-m_{2} m_{3} m_{23}-m_{1} m_{3} m_{13}$.

(This appears in the book [14] of Fricke and Klein.) That there is precisely one relation fits nicely with the rough dimension count of $3 \cdot 3-3=6$ for the space of conjugacy classes of triples. Viewed as a quadratic equation for $m_{321}$ the other root of (10) is $\operatorname{Tr}\left(M_{1} M_{2} M_{3}\right)$ so in particular we have

$$
\operatorname{Tr}\left(M_{1} M_{2} M_{3}\right)=P-m_{321}
$$

Note that, upon fixing $m_{1}, m_{2}, m_{3}, m_{321}$, the Fricke relation is a cubic equation in the remaining three variables; the six dimensional variety we are studying is essentially a universal family of affine cubic surfaces [22].

Now, the induced $\mathcal{B}_{3}$ action on conjugacy classes of triples induces an action on the invariant functions, and we will describe this action in terms of the seven chosen generators. Clearly $m_{321}$ is fixed by both $\beta_{i}$, and the $m_{i}$ are just permuted:

$$
\beta_{1}\left(m_{1}, m_{2}, m_{3}\right)=\left(m_{1}, m_{3}, m_{2}\right), \quad \beta_{2}\left(m_{1}, m_{2}, m_{3}\right)=\left(m_{2}, m_{1}, m_{3}\right) .
$$

Lemma 1. The induced $\mathcal{B}_{3}$ action on the quadratic functions is

$$
\begin{aligned}
& \beta_{1}\left(m_{12}, m_{23}, m_{13}\right)=\left(m_{2} m_{321}+m_{1} m_{3}-m_{13}-m_{12} m_{23}, m_{23}, m_{12}\right) \\
& \beta_{2}\left(m_{12}, m_{23}, m_{13}\right)=\left(m_{12}, m_{13}, m_{1} m_{321}+m_{2} m_{3}-m_{23}-m_{13} m_{12}\right)
\end{aligned}
$$


Proof. The second formula follows from the first by permuting indices. For the first formula the hard part is to establish

$$
\operatorname{Tr}\left(M_{2}^{-1} M_{3} M_{2} M_{1}\right)=m_{2} m_{321}+m_{1} m_{3}-m_{13}-m_{12} m_{23} .
$$

One way to do this (which will extend to the $3 \times 3$ case below) is to write $M_{i}=\varepsilon_{i}\left(1+e_{i} \otimes \alpha_{i}\right)$ for some rank one matrix $e_{i} \otimes \alpha_{i}$ and number $\varepsilon_{i} \in \mathbb{C}^{*}$. Then $\operatorname{Tr}\left(M_{2}^{-1} M_{3} M_{2} M_{1}\right)$ can be expanded in terms of the numbers $\alpha_{i}\left(e_{j}\right)$ and the terms of the resulting expression can be identified as terms in the expansions of the seven invariant functions.

Before moving on to the higher rank case we point out the evident fact that if $\left(M_{3}, M_{2}, M_{1}\right)$ are a triple of generators of a finite subgroup of $G$ then the corresponding braid group orbit is finite (and in turn the induced action on conjugacy classes of triples is also finite).

\section{$3 \times 3$ case.}

Now we wish to find analogous formulae for the corresponding action of $\mathcal{B}_{3}$ on conjugacy classes of triples of pseudo-reflections in $\mathrm{GL}_{3}(\mathbb{C})$.

Suppose $r_{1}, r_{2}, r_{3}$ are pseudo-reflections in $\mathrm{GL}_{3}(\mathbb{C})$, so that

$$
r_{i}=1+e_{i} \otimes \alpha_{i}
$$

for some $e_{i} \in V, \alpha_{i} \in V^{*}$ where $V=\mathbb{C}^{3}$. Choose six non-zero complex numbers $n_{1}, n_{2}, n_{3}, t_{1}, t_{2}, t_{3}$ such that $t_{i}$ is a choice of square root of $\operatorname{det}\left(r_{i}\right)$ (i.e. $t_{i}^{2}=1+\alpha_{i}\left(e_{i}\right)$ ), that the product $r_{3} r_{2} r_{1}$ has eigenvalues $\left\{n_{1}^{2}, n_{2}^{2}, n_{3}^{2}\right\}$ and that these square roots are chosen so that

$$
t_{1} t_{2} t_{3}=n_{1} n_{2} n_{3}
$$

(which is a square root of the equation $\left.\prod\left(\operatorname{det} r_{i}\right)=\operatorname{det} r_{3} r_{2} r_{1}\right)$. These square roots (and the choice of ordering of eigenvalues of $r_{3} r_{2} r_{1}$ ) will not be needed to describe the braid group actions here, but will be convenient later.

Now consider the following eight $\mathrm{GL}_{3}(\mathbb{C})$-invariant functions on the set of triples of pseudo-reflections:

$$
\begin{gathered}
t_{12}:=\operatorname{Tr}\left(r_{1} r_{2}\right)-1, \quad t_{23}:=\operatorname{Tr}\left(r_{2} r_{3}\right)-1, \quad t_{13}:=\operatorname{Tr}\left(r_{1} r_{3}\right)-1, \\
t_{321}:=n_{1}^{2}+n_{2}^{2}+n_{3}^{2}, \quad t_{321}^{\prime}:=\left(n_{1} n_{2}\right)^{2}+\left(n_{2} n_{3}\right)^{2}+\left(n_{1} n_{3}\right)^{2} .
\end{gathered}
$$

(Note that $t_{i}^{2}=\operatorname{Tr}\left(r_{i}\right)-2, t_{321}:=\operatorname{Tr}\left(r_{3} r_{2} r_{1}\right)$ and $t_{321}^{\prime}=\operatorname{det}\left(r_{3} r_{2} r_{1}\right) \operatorname{Tr}\left(r_{3} r_{2} r_{1}\right)^{-1}$.) The subtractions of 1 or 2 in this definition turn out to simplify the formulae below. The action of $\mathcal{B}_{3}$ on triples of pseudo-reflections is generated by

$$
\begin{aligned}
& \beta_{1}\left(r_{3}, r_{2}, r_{1}\right)=\left(r_{2}, r_{2}^{-1} r_{3} r_{2}, r_{1}\right), \\
& \beta_{2}\left(r_{3}, r_{2}, r_{1}\right)=\left(r_{3}, r_{1}, r_{1}^{-1} r_{2} r_{1}\right) .
\end{aligned}
$$

Now consider the induced action on conjugacy classes of triples. First, it is clear that $t_{321}, t_{321}^{\prime}$ are $\mathcal{B}_{3}$-invariant since $r_{3} r_{2} r_{1}$ is fixed. Also, as before, the functions $t_{i}^{2}$ are just permuted:

$$
\beta_{1}\left(t_{1}^{2}, t_{2}^{2}, t_{3}^{2}\right)=\left(t_{1}^{2}, t_{3}^{2}, t_{2}^{2}\right), \quad \beta_{2}\left(t_{1}^{2}, t_{2}^{2}, t_{3}^{2}\right)=\left(t_{2}^{2}, t_{1}^{2}, t_{3}^{2}\right)
$$


Lemma 2. The induced $\mathcal{B}_{3}$ action on the functions $\left(t_{12}, t_{23}, t_{13}\right)$ is as follows:

$$
\begin{aligned}
& \beta_{1}\left(t_{12}, t_{23}, t_{13}\right)=\left(t_{321}+t_{1}^{2}+t_{3}^{2}-t_{13}+\left(t_{321}^{\prime}-t_{12} t_{23}\right) / t_{2}^{2}, t_{23}, t_{12}\right) \\
& \beta_{2}\left(t_{12}, t_{23}, t_{13}\right)=\left(t_{13}, t_{23}, t_{321}+t_{2}^{2}+t_{3}^{2}-t_{23}+\left(t_{321}^{\prime}-t_{13} t_{12}\right) / t_{1}^{2}\right) .
\end{aligned}
$$

Proof. The non-obvious part is to establish

$$
\operatorname{Tr}\left(r_{2}^{-1} r_{3} r_{2} r_{1}\right)=t_{321}+t_{1}^{2}+t_{3}^{2}-t_{13}+\left(t_{321}^{\prime}-t_{12} t_{23}\right) / t_{2}^{2} .
$$

For this we first observe $r_{i}^{-1}=1-e_{i} \otimes \alpha_{i} / t_{i}^{2}$. Then expanding $\operatorname{Tr}\left(r_{2}^{-1} r_{3} r_{2} r_{1}\right)$ yields

$$
t_{321}+1-t_{2}^{2}-u_{12} u_{21}-u_{23} u_{32}-\left(u_{12} u_{23} u_{31}+u_{23} u_{32} u_{12} u_{21}\right) / t_{2}^{2}
$$

where $u_{i j}:=\alpha_{i}\left(e_{j}\right)$. To simplify this we first use the following identities (obtained by expanding the traces $\left.t_{i j}\right)$ :

$$
u_{i j} u_{j i}=t_{i j}-t_{i}^{2}-t_{j}^{2} \quad \text { if } i \neq j .
$$

Then, to finish, we use the identity (analogous to (11)):

$$
u_{12} u_{23} u_{31}=t_{3}^{2} t_{12}+t_{2}^{2} t_{13}+t_{1}^{2} t_{23}-\left(t_{1} t_{2}\right)^{2}-\left(t_{2} t_{3}\right)^{2}-\left(t_{1} t_{3}\right)^{2}-t_{321}^{\prime},
$$

which is obtained by expanding $\operatorname{Tr}\left(r_{1}^{-1} r_{2}^{-1} r_{3}^{-1}\right)=\operatorname{Tr}\left(\left(r_{3} r_{2} r_{1}\right)^{-1}\right)=n_{1}^{-2}+n_{2}^{-2}+n_{3}^{-2}$.

Again we have the evident fact that if $\left(r_{3}, r_{2}, r_{1}\right)$ are a triple of generators of a finite subgroup of $\mathrm{GL}_{3}(\mathbb{C})$, i.e. if they are generators of a three-dimensional complex reflection group, then the corresponding braid group orbit is finite (and in turn the induced action on conjugacy classes of triples is also finite).

Remark 3. A rough dimension count gives $3 \cdot 5-8=7$ for the space of conjugacy classes of pseudo-reflections, so we expect there to be a relation amongst the eight invariant functions. This is the analogue of the Fricke relation and comes from the identity

$$
\left(u_{12} u_{23} u_{31}\right)\left(u_{32} u_{21} u_{13}\right)=\left(u_{23} u_{32}\right)\left(u_{12} u_{21}\right)\left(u_{13} u_{31}\right) .
$$

Rewriting each bracketed term in terms of the eight functions yields the desired relation:

$$
\begin{gathered}
\left(t_{3}^{2} t_{12}+t_{2}^{2} t_{13}+t_{1}^{2} t_{23}-\left(t_{1} t_{2}\right)^{2}-\left(t_{2} t_{3}\right)^{2}-\left(t_{1} t_{3}\right)^{2}-t_{321}^{\prime}\right) \\
\times\left(t_{321}+t_{1}^{2}+t_{2}^{2}+t_{3}^{2}-t_{12}-t_{13}-t_{23}\right) \\
=\left(t_{12}-t_{1}^{2}-t_{2}^{2}\right)\left(t_{13}-t_{1}^{2}-t_{3}^{2}\right)\left(t_{23}-t_{2}^{2}-t_{3}^{2}\right) .
\end{gathered}
$$

\section{From $3 \times 3$ to $2 \times 2$.}

Now we will define a $\mathcal{B}_{3}$-equivariant map from the space of triples of pseudo-reflections to the space of $\mathrm{SL}_{2}(\mathbb{C})$ triples. The main application of this here is just the observation that we will then obtain more exotic finite $\mathrm{SL}_{2}(\mathbb{C})$ braid group orbits from any triple of generators of a complex reflection group.

Suppose we are given the data

$$
\mathbf{t}:=\left(t_{1}, t_{2}, t_{3}, n_{1}, n_{2}, n_{3}, t_{12}, t_{23}, t_{13}\right)
$$

associated to a triple of pseudo-reflections. (We extend the $\mathcal{B}_{3}$-action to the set of such data -i.e. with square root choices etc. - in the obvious way, permuting the $t_{i}$ and fixing the $\left.n_{i}.\right)$ Define a map $\varphi$ taking $\mathbf{t}$ to the $\mathrm{SL}_{2}(\mathbb{C})$ data $\mathbf{m}$ given by: 


$$
\begin{aligned}
& m_{1}:=\frac{t_{1}}{n_{1}}+\frac{n_{1}}{t_{1}}, \quad m_{2}:=\frac{t_{2}}{n_{1}}+\frac{n_{1}}{t_{2}}, \quad m_{3}:=\frac{t_{3}}{n_{1}}+\frac{n_{1}}{t_{3}}, \\
& m_{12}:=\frac{t_{12}}{t_{1} t_{2}}, \quad m_{23}:=\frac{t_{23}}{t_{2} t_{3}}, \quad m_{13}:=\frac{t_{13}}{t_{1} t_{3}}, \\
& m_{321}:=\frac{n_{2}}{n_{3}}+\frac{n_{3}}{n_{2}} .
\end{aligned}
$$

Theorem 1. The map $\varphi$ is $\mathcal{B}_{3}$-equivariant. In particular finite $\mathcal{B}_{3}$-orbits of $\mathrm{SL}_{2}(\mathbb{C})$ triples are obtained from triples of generators of three-dimensional complex reflection groups.

Proof. This may be proved by direct calculation (a less direct proof will be given in section [3, along with a description of the origins of the above formulae). For example if we write $\mathbf{m}^{\prime}=\beta_{1}(\varphi(\mathbf{t}))$ and $\mathbf{m}^{\prime \prime}=\varphi\left(\beta_{1}(\mathbf{t})\right)$, then the tricky part is to see $m_{12}^{\prime}=m_{12}^{\prime \prime}$. However it is straightforward to show that the expression obtained for $m_{12}^{\prime}-m_{12}^{\prime \prime}$ (using the above formulae) has a factor of $t_{1} t_{2} t_{3}-n_{1} n_{2} n_{3}$ in its numerator, which is zero due to (12). (Similarly for $\beta_{2}$.)

Remark 4. It is possible to check directly (using Maple) that the map $\varphi$ is well-defined; i.e. that $\varphi(\mathbf{t})$ satisfies the Fricke relation (10) provided that $\mathbf{t}$ satisfies both (12) and (16)).

\section{Painlevé parameters.}

The Painlevé VI equation that arises by performing isomonodromic deformations of the rank 2 Fuchsian system with monodromy data $M_{1}, M_{2}, M_{3}, M_{4}$ (where $M_{4} M_{3} M_{2} M_{1}=1$ ) has parameters

$$
\alpha=\left(\theta_{4}-1\right)^{2} / 2, \beta=-\theta_{1}^{2} / 2, \gamma=\theta_{3}^{2} / 2, \delta=\left(1-\theta_{2}^{2}\right) / 2
$$

where the $\theta_{j}(j=1,2,3,4)$ are such that $M_{j}$ has eigenvalues $\exp \left( \pm \pi i \theta_{j}\right)$, i.e.

$$
\operatorname{Tr}\left(M_{j}\right)=2 \cos \left(\pi \theta_{j}\right) .
$$

Now suppose the $M_{j}$ arise under the map $\varphi$ from some data $\mathbf{t}$ associated to a threedimensional pseudo-reflection group. We can then relate the Painlevé parameters to the invariants of the pseudo-reflection group (cf. 6] Lemma 3). If we choose (for $j=1,2,3$ ) $\lambda_{j}, \mu_{j}$ such that

$$
t_{j}=\exp \left(\pi i \lambda_{j}\right), \quad n_{j}=\exp \left(\pi i \mu_{j}\right), \quad \sum \lambda_{i}=\sum \mu_{i}
$$

then we have:

Lemma 5. The Painlevé parameters corresponding to the data $\mathbf{t}$ under the map $\varphi$ are

$$
\theta_{i}=\lambda_{i}-\mu_{1}(i=1,2,3), \quad \theta_{4}=\mu_{3}-\mu_{2} .
$$

Proof. From the definition (17) of $\varphi$ we have $\operatorname{Tr}\left(M_{i}\right)=m_{i}=2 \cos \pi\left(\lambda_{i}-\mu_{1}\right)$ and $\operatorname{Tr}\left(M_{4}\right)=m_{321}=2 \cos \pi\left(\mu_{3}-\mu_{2}\right)$.

In particular if we are considering a complex reflection group $G$ that is generated by a triple of reflections, then, by 5.4 of [31], we may choose a generating triple $\left(r_{1}, r_{2}, r_{3}\right)$ such that the $\mu_{i}$ are related to the exponents $x_{1} \leqslant x_{2} \leqslant x_{3}$ of the group $G$ as follows:

$$
\mu_{i}=x_{i} / h, \quad(i=1,2,3) \quad h:=x_{3}+1 .
$$


This result enables us to compile a table of parameters of the Painlevé equations corresponding to the standard generators of those irreducible three-dimensional complex reflection groups which may be generated by three reflections (see [6] Table 1, p.1019).

Example. Let us consider the reflections generating the Klein complex reflection group. Explicitly the standard generators (from 31] 10.1) are:

$$
r_{1}=\frac{1}{2}\left(\begin{array}{ccc}
1 & -1 & -\bar{a} \\
-1 & 1 & -\bar{a} \\
-a & -a & 0
\end{array}\right), \quad r_{2}=\left(\begin{array}{ccc}
1 & 0 & 0 \\
0 & 1 & 0 \\
0 & 0 & -1
\end{array}\right), \quad r_{3}=\left(\begin{array}{ccc}
1 & 0 & 0 \\
0 & 0 & 1 \\
0 & 1 & 0
\end{array}\right)
$$

where $a:=(1+i \sqrt{7}) / 2$. These are order two complex reflections so $\operatorname{det}\left(r_{i}\right)=-1$ and are ordered so that the exponents $\{3,5,13\}$ of the group appear in the eigenvalues of the product $r_{3} r_{2} r_{1}$. Namely $r_{3} r_{2} r_{1}$ has eigenvalues $\left\{\exp \left(2 \pi i \frac{3}{14}\right), \exp \left(2 \pi i \frac{5}{14}\right), \exp \left(2 \pi i \frac{13}{14}\right)\right\}$. Also we compute:

$$
\operatorname{Tr}\left(r_{1} r_{2}\right)=1, \quad \operatorname{Tr}\left(r_{2} r_{3}\right)=1, \quad \operatorname{Tr}\left(r_{1} r_{3}\right)=0 .
$$

Thus if we set $\lambda_{1}=\lambda_{2}=\lambda_{3}=1 / 2, \mu_{1}=3 / 14, \mu_{2}=5 / 14, \mu_{3}=13 / 14$ so that

$$
t_{1}=t_{2}=t_{3}=i, \quad n_{1}=\exp \frac{3 \pi i}{14}, \quad n_{2}=\exp \frac{5 \pi i}{14}, \quad n_{3}=\exp \frac{13 \pi i}{14}
$$

then the image of this data under $\varphi$ is

$$
m_{1}=m_{2}=m_{3}=2 \cos (2 \pi / 7), \quad m_{321}=2 \cos (4 \pi / 7), \quad m_{12}=m_{23}=0, \quad m_{13}=1 .
$$

Clearly (cf. Lemma 5) the parameters of the corresponding Painlevé equation are:

$$
\theta_{1}=\theta_{2}=\theta_{3}=2 / 7, \quad \theta_{4}=4 / 7 \quad \text { and so } \quad(\alpha, \beta, \gamma, \delta)=(9,-4,4,45) / 98 .
$$

The corresponding braid group orbit is easy to calculate by hand; Observe each of $m_{1}, m_{2}, m_{3}, m_{321}$ is fixed by $\mathcal{B}_{3}$, and that, since $4 \cos (2 \pi / 7) \cos (4 \pi / 7)+4 \cos ^{2}(2 \pi / 7)=1$, the formula for the action on the quadratic functions simplifies to

$$
\begin{aligned}
& \beta_{1}\left(m_{12}, m_{23}, m_{13}\right)=\left(1-m_{13}-m_{12} m_{23}, m_{23}, m_{12}\right), \\
& \beta_{2}\left(m_{12}, m_{23}, m_{13}\right)=\left(m_{12}, m_{13}, 1-m_{23}-m_{13} m_{12}\right) .
\end{aligned}
$$

In this way we find the $\mathcal{B}_{3}$ orbit has size seven, with values

$\begin{array}{ccc}m_{12} & m_{23} & m_{13} \\ 0 & 0 & 0 \\ 0 & 0 & 1 \\ 0 & 1 & 0 \\ 0 & 1 & 1 \\ 1 & 0 & 0 \\ 1 & 0 & 1 \\ 1 & 1 & 0 .\end{array}$

Upon restriction to the pure subgroup $\mathcal{P}_{3} \subset \mathcal{B}_{3}$, whose action is generated by $\beta_{1}^{2}, \beta_{2}^{2}$, we find the orbit still has size seven. (More precisely, $\beta_{1}^{2}, \beta_{2}^{2}$ generate the free subgroup $\mathcal{F}_{2} \subset \mathcal{P}_{3}$, and $\mathcal{P}_{3}$ is generated by $\mathcal{F}_{2}$ and its centre, which acts trivially.) Thus the corresponding solution of Painlevé VI has seven branches and, via (21), the branches of the solution are conveniently labelled by the (binary) numbers from zero to six. The 
generators of the pure braid group action are represented by the following permutations of the seven branches:

$$
\begin{array}{ll}
\beta_{1}^{2}: & (05)(14)(236) \\
\beta_{2}^{2}: & \quad(03)(12)(465)
\end{array}
$$

whose product also has two 2-cycles and a 3-cycle. This should be the monodromy representation of the solution curve as a cover of $\mathbb{P}^{1}$ branched at $0,1, \infty$. Using the RiemannHurwitz formula we thus see the solution curve has genus zero. Also, for example, one can calculate the monodromy group of the cover; the subgroup of the symmetric group generated by these (even) permutations is as large as possible, namely $A_{7}$. This gives a clear picture of the solution curve topologically as a cover of the Riemann sphere. Our next aim (after explaining how the formulae of this section were found) will be to find an explicit polynomial equation for the solution curve and for the function $y$ on it solving Painlevé VI.

Remark 6. Given the data corresponding to any of these branches one can easily solve the seven equations (9) to find a corresponding $\mathrm{SL}_{2}(\mathbb{C})$ triple. For example for branch zero it is straightforward to find the triple:

$$
M_{1}=\left(\begin{array}{cc}
\phi & 0 \\
0 & \phi^{-1}
\end{array}\right), \quad M_{2}=\left(\begin{array}{cc}
w & x \\
-x & \bar{w}
\end{array}\right), \quad M_{3}=\left(\begin{array}{cc}
w & \mu x \\
-x / \mu & \bar{w}
\end{array}\right),
$$

where $\phi=e^{2 \pi i / 7}, w=\frac{1+\phi^{2}}{\phi-\phi^{3}}, x=\sqrt{1-|w|^{2}} \in \mathbb{R}_{>0}$ and $\mu=\left(r+i \sqrt{4-r^{2}}\right) / 2$ where $r=\frac{\left(1+\phi^{2}\right)^{2}}{1-\phi^{2}} \in[0,1] \subset \mathbb{R}$.

Lemma 7. The group generated by $M_{1}, M_{2}, M_{3}$ is an infinite subgroup of $\mathrm{SU}_{2}$.

Proof. By construction the $M_{i}$ are in $\mathrm{SU}_{2}$ (somewhat surprisingly). Since the group is nonabelian and contains elements of order seven we are still to check it is not some large dihedral group. Let $\varepsilon$ be an eigenvalue of $M_{1}^{4} M_{2}$, so that $\varepsilon+\varepsilon^{-1}=\tau$ where $\tau=-\left(1+\phi^{2}\right)\left(\phi+\phi^{4}+\phi^{6}\right)$ and so $\varepsilon=\left(\tau \pm \sqrt{\tau^{2}-4}\right) / 2$. Thus $\varepsilon$ is some algebraic number of modulus one and we claim it is not a root of unity. To see this we take the product of $z-\varepsilon^{\prime}$ over all the Galois conjugates of $\varepsilon$ and find the minimal polynomial of $\varepsilon$ is

$$
p(z)=z^{6}-3 z^{5}-z^{4}-7 z^{3}-z^{2}-3 z+1 .
$$

Thus if $\varepsilon^{N}=1$ for some integer $N$ we would have that $p$ divides $z^{N}-1$ so all the roots of $p$ are roots of unity. However $p(1)=-13$ so $p$ has a real root greater than 1 .

(Note also that triples in the same braid group orbit generate the same group.)

\section{Some properties of $\varphi$.}

Before moving on to the Fourier-Laplace transform we will describe some properties of the map $\varphi$; we will show that the different choices of square roots etc. give isomorphic $\mathcal{P}_{3}$ orbits and also examine the fibres of $\varphi$. This motivates the definition of $\varphi$.

First of all, the conjugacy class of a triple of pseudo-reflections only determines the values of the functions $t_{1}^{2}, t_{2}^{2}, t_{3}^{2}, t_{12}, t_{23}, t_{13}$ and the symmetric functions in the $n_{i}^{2}$, whereas the map $\varphi$ involves each $t_{i}, n_{i}$. Thus we must make a choice of ordering of the eigenvalues $n_{i}^{2}$ of $r_{3} r_{2} r_{1}$ and of the square roots $t_{i}, n_{i}$ such that $t_{1} t_{2} t_{3}=n_{1} n_{2} n_{3}$ in order to obtain the $2 \times 2$ data. In general different choices lead to different $2 \times 2$ data (cf. Remark 14). However the pure braid group orbits obtained via different choices are all isomorphic: 
Lemma 8. Let $\pi$ be a permutation of $\{1,2,3\}$ and choose signs $\varepsilon_{i}, \delta_{i} \in\{ \pm 1\}$ for $i=1,2,3$ such that $\varepsilon_{1} \varepsilon_{2} \varepsilon_{3}=\delta_{1} \delta_{2} \delta_{3}$. Consider the map $\sigma$ on the set of data

$$
\mathbf{t}=\left(t_{1}, t_{2}, t_{3}, n_{1}, n_{2}, n_{3}, t_{12}, t_{23}, t_{13}\right)
$$

(satisfying (12) and (16) ) defined by

$$
\sigma(\mathbf{t}):=\left(\varepsilon_{1} t_{1}, \varepsilon_{2} t_{2}, \varepsilon_{3} t_{3}, \delta_{1} n_{\pi(1)}, \delta_{2} n_{\pi(2)}, \delta_{3} n_{\pi(3)}, t_{12}, t_{23}, t_{13}\right) .
$$

Then map $\sigma$ commutes with the action of the pure braid group $\mathcal{P}_{3} \subset \mathcal{B}_{3}$, and in particular the $\mathcal{P}_{3}$ orbits through $\varphi(\mathbf{t})$ and $\varphi(\sigma(\mathbf{t}))$ are isomorphic.

Proof. The pure braid group action is generated by $\beta_{1}^{2}, \beta_{2}^{2}$ and so fixes the $t_{i}, n_{i}$ pointwise. From Lemma 2 the action on the functions $\left(t_{12}, t_{23}, t_{13}\right)$ is independent of the sign and ordering choices.

We also wish to check that the different possible sign/ordering choices lead to Painlevé VI equations with parameters which are equivalent under the action of the affine $F_{4}$ Weyl group symmetries defined by Okamoto [30]. To this end we lift the map $\sigma$ to act on the data $\Lambda:=\left(\lambda_{1}, \lambda_{2}, \lambda_{3}, \mu_{1}, \mu_{2}, \mu_{3}\right)$ of (19) as

$$
\sigma(\boldsymbol{\Lambda})=\left(\lambda_{1}+a_{1}, \lambda_{2}+a_{2}, \lambda_{3}+a_{3}, \mu_{\pi(1)}+b_{1}, \mu_{\pi(2)}+b_{2}, \mu_{\pi(3)}+b_{3}\right)
$$

where $a_{i}, b_{i}$ are integers such that $\sum a_{i}=\sum b_{i}$ and $\pi$ is a permutation of $\{1,2,3\}$.

Lemma 9. The Painlevé VI parameters associated to $\boldsymbol{\Lambda}=\left(\lambda_{1}, \lambda_{2}, \lambda_{3}, \mu_{1}, \mu_{2}, \mu_{3}\right)$ in Lemma 5 are equivalent, under Okamoto's affine $F_{4}$ Weyl group action, to those associated to $\sigma(\boldsymbol{\Lambda})$.

Proof. Since the set of such $\sigma$ 's forms a group it is sufficient to check the lemma on generators. The translations and the permutations may be dealt with separately since the group is a semi-direct product. First for the translations (fixing $\pi$ to be the trivial permutation) this is straightforward; for example it is easy to express the corresponding translations of the $\theta$ 's in terms of the translations of [29] (34). Finally the permutation just swapping $\mu_{1}$ and $\mu_{2}$ is obtained from the transformation $s_{1} s_{2} s_{1}$ (of [29]) and that swapping $\mu_{1}$ and $\mu_{3}$ is obtained from the transformation $\left(s_{0} s_{1} s_{3} s_{4}\right) s_{2}\left(s_{0} s_{1} s_{3} s_{4}\right)$.

Remark 10. Perhaps it is helpful to recall that there are several symmetry groups of PVI considered in the literature, amongst which we have

$$
\text { affine } D_{4}<\text { extended affine } D_{4}<\text { affine } F_{4}
$$

the first two of which are for example considered in [29]. In brief ${ }^{3}$ the first two differ by the Klein four-group and do not involve changing the time parameter $t$, whereas the full affine $F_{4}$ action of Okamoto involves changing $t$ (by automorphisms of $\mathbb{P}^{1}$ permuting $0,1, \infty)$. In fact only the extended affine $D_{4}$ symmetries were used above, although the full $F_{4}$ action will be considered in section 6 .

\footnotetext{
${ }^{3}$ I am grateful to M. Noumi for clarifying this to me.
} 
Next we will examine the fibres of $\varphi$. From our rough dimension counts we see these fibres should be one dimensional. The continuous part of the fibres arises as follows. Define an action of $\mathbb{C}^{*}$ on the pseudo-reflection data $\{\mathbf{t}\}$ by declaring $h \in \mathbb{C}^{*}$ to act as

$$
t_{i} \mapsto h t_{i}, \quad n_{i} \mapsto h n_{i}, \quad t_{i j} \mapsto h^{2} t_{i j}
$$

Observe that this does indeed act within the fibres of $\varphi$, i.e. that $\varphi(h \mathbf{t})=\varphi(\mathbf{t})$ for any $h \in \mathbb{C}^{*}$. Moreover a simple direct calculation shows this $\mathbb{C}^{*}$ action commutes with the $\mathcal{B}_{3}$ action on $\{\mathbf{t}\}$. (The simplicity of this action is deceptive since we carefully chose the functions $t_{i}, t_{i j}$.)

Thus, for example, we can always use this action to move to the ( $\mathcal{B}_{3}$-invariant) subset of the pseudo-reflection data having $n_{1}=1$.

Lemma 11. The map $\varphi$ is surjective, and the restriction of $\varphi$ to the subset of the pseudoreflection data $\{\mathbf{t}\}$ having $n_{1}=1$ is a finite map.

Proof. Given arbitrary $\mathrm{SL}_{2}(\mathbb{C})$ data $\mathbf{m}$ we just try to solve for $\mathbf{t}$ (having first set $n_{1}=1$ ). One finds a solution always exists and there are five sign choices, so a generic fibre has 32 points.

Remark 12. One may check algebraically that if $\mathbf{t}$ has $n_{1}=1$, satisfies $n_{2} n_{3}=t_{1} t_{2} t_{3}$ and is such that $\varphi(\mathbf{t})$ satisfies the Fricke relation (10) then $\mathbf{t}$ satisfies the $3 \times 3$ analogue (16) of the Fricke relation.

Moving to the subset of the data on which $n_{1}=1$ implies we are forcing 1 into the spectrum of the product $r_{3} r_{2} r_{1}$. This implies that the representation (of the free group on three letters) defined by $\left(r_{3}, r_{2}, r_{1}\right)$ is reducible: This is clear if $r_{i}=1+e_{i} \otimes \alpha_{i}$ for some $e_{i}$ which are not a basis of $\mathbb{C}^{3}$ (since the span of the $e_{i}$ is an invariant subspace). Otherwise we have:

Lemma 13 (cf. [7] 10.5.6, 8] 3.7). If $r_{i}:=1+e_{i} \otimes \alpha_{i}$ for a basis $e_{1}, e_{2}, e_{3}$ of $V=\mathbb{C}^{3}$ and $v \in V$ satisfies $r_{3} r_{2} r_{1} v=v$ then $r_{i} v=v$ for $i=1,2,3$.

Proof. If $r_{3} r_{2} r_{1} v=v$ then

$$
r_{2} r_{1} v-v=r_{3}^{-1} v-v
$$

the lefthand side of which is a linear combination of $e_{2}, e_{1}$, and the righthand side is a multiple of $e_{3}$, since $r_{3}^{-1}=1-e_{3} \otimes \alpha_{3} / t_{3}^{2}$. Thus both sides vanish so $r_{3} v=v$ and $r_{2} r_{1} v=v$. Then similarly we see both sides $r_{1} v-v=r_{2}^{-1} v-v$ vanish.

Thus we can use the $\mathbb{C}^{*}$ action to move to a reducible triple. The map $\varphi$ is defined simply by first moving to a reducible triple by setting $h:=n_{1}^{-1}$ so that we can write the $r_{i}$ in block upper triangular form. In general there will then be a size two and a size one block (with entry 1 ) on the diagonal. We then define $\widehat{M}_{i} \in \mathrm{GL}_{2}(\mathbb{C})$ to be the size two block, which will have eigenvalues $\left\{1,\left(t_{i} / n_{1}\right)^{2}\right\}$. Hence defining $M_{i}=n_{1} \widehat{M}_{i} / t_{i}$ yields an $\mathrm{SL}_{2}(\mathbb{C})$ triple. Computing the various traces then gives the stated formulae for the map $\varphi$. (In more invariant language we take the projection to $\mathrm{SL}_{2}(\mathbb{C})$ of the rank two part of the 'semisimplification' of the reducible representation.)

Thus we have motivated the map $\varphi$ in terms of the $\mathbb{C}^{*}$ action. In section 3 we will motivate this action as the image under the Fourier-Laplace transform of a simple scalar shift. 


\section{Orthogonal reflection groups.}

Let us check that the unipotent $2 \times 2$ monodromy data and the orthogonal threedimensional reflection groups considered by Dubrovin-Mazzocco in [13] are related by the $\operatorname{map} \varphi$ defined in (17).

The monodromy data in 13 is parameterised by four numbers $\left(x_{1}, x_{2}, x_{3}, \mu\right)$ related by the condition

$$
m=2 \cos (2 \pi \mu) \quad \text { where } m:=2+x_{1} x_{2} x_{3}-\left(x_{1}^{2}+x_{2}^{2}+x_{3}^{2}\right),
$$

which is equivalent to [13] (1.21). The degenerate cases $m= \pm 2$ are ruled out. Although the main interest is in real orthogonal reflection groups the formulae here make sense for complex values of the parameters; effectively we are restricting $\varphi$ to a complex threedimensional slice.

Without loss of generality one may assume $x_{1} \neq 0$ and then the $2 \times 2$ monodromy data is given by the triple $([13](1.20))$ :

$$
M_{1}=\left(\begin{array}{cc}
1 & -x_{1} \\
0 & 1
\end{array}\right), \quad M_{2}=\left(\begin{array}{cc}
1 & 0 \\
x_{1} & 1
\end{array}\right), \quad M_{3}=\left(\begin{array}{cc}
1+x_{2} x_{3} / x_{1} & -x_{2}^{2} / x_{1} \\
x_{3}^{2} / x_{1} & 1-x_{2} x_{3} / x_{1}
\end{array}\right) .
$$

Note that, generically, each of these matrices is conjugate to $\left(\begin{array}{ll}1 & 1 \\ 0 & 1\end{array}\right)$, and in all cases $m_{j}:=$ $\operatorname{Tr}\left(M_{j}\right)=2$. Also, straightforward computations give that

$$
m_{12}=2-x_{1}^{2}, \quad m_{23}=2-x_{2}^{2}, \quad m_{13}=2-x_{3}^{2}, \quad m_{321}=m .
$$

(By nondegeneracy there is always some index $j$ such that $x_{j} \neq 0$ and one may obtain the same values of the invariant functions from an analogous $2 \times 2$ triple.)

The corresponding $3 \times 3$ reflections considered in [13] are $(13$ (1.51)):

$$
r_{1}=\left(\begin{array}{ccc}
-1 & -x_{1} & -x_{3} \\
0 & 1 & 0 \\
0 & 0 & 1
\end{array}\right), \quad r_{2}=\left(\begin{array}{ccc}
1 & 0 & 0 \\
-x_{1} & -1 & -x_{2} \\
0 & 0 & 1
\end{array}\right), \quad r_{3}=\left(\begin{array}{ccc}
1 & 0 & 0 \\
0 & 1 & 0 \\
-x_{3} & -x_{2} & -1
\end{array}\right)
$$

which preserve the nondegenerate symmetric bilinear form given by the matrix

$$
\left(\begin{array}{ccc}
2 & x_{1} & x_{3} \\
x_{1} & 2 & x_{2} \\
x_{3} & x_{2} & 2
\end{array}\right)
$$

Immediate computation then gives that

$$
\begin{gathered}
t_{1}^{2}=t_{2}^{2}=t_{3}^{2}=-1, \\
t_{12}=x_{1}^{2}-2, \quad t_{23}=x_{2}^{2}-2, \quad t_{13}=x_{3}^{2}-2 \\
t_{321}=-m-1=-t_{321}^{\prime} .
\end{gathered}
$$

The characteristic polynomial of $r_{3} r_{2} r_{1}$ is

$$
(\lambda+1)\left(\lambda^{2}+m \lambda+1\right)
$$

which has roots

$$
n_{1}^{2}=-1, \quad n_{2}^{2}=-\exp (2 \pi i \mu), \quad n_{3}^{2}=-\exp (-2 \pi i \mu) .
$$

Now we claim that if we choose the square roots appropriately then the map $\varphi$ of (17) takes this $3 \times 3$ data onto the unipotent $2 \times 2$ data above. Indeed setting $n_{1}=t_{1}=$ $t_{2}=t_{3}=i$ clearly gives the correct values $m_{j}=i / i+i / i=2$ and $m_{j k}=t_{j k} / i^{2}=-t_{j k}$. Also if we set $n_{2}=i \exp (\pi i \mu)$ and $n_{3}=i \exp (-\pi i \mu)$ then $n_{2} / n_{3}=\exp (2 \pi i \mu)$ and so 
$m_{321}=n_{2} / n_{3}+n_{3} / n_{2}=2 \cos 2 \pi \mu=m$ as required. Thus the map $\varphi$ does indeed extend the above correspondence used by Dubrovin-Mazzocco.

Remark 14. If instead we choose to order the eigenvalues of $r_{3} r_{2} r_{1}$ as

$$
n_{1}^{2}=-\exp (2 \pi i \mu), \quad n_{2}^{2}=-1, \quad n_{3}^{2}=-\exp (-2 \pi i \mu)
$$

then we claim that, with appropriate square root choices, the corresponding $2 \times 2$ data (un$\operatorname{der} \varphi$ ) has the remarkable property that the four local monodromies $M_{1}, M_{2}, M_{3}, M_{3} M_{2} M_{1}$ all lie in the same conjugacy class: Namely if we choose $n_{2}=t_{1}=t_{2}=t_{3}=i$, let $n_{1}$ be any square root of $-\exp (2 \pi i \mu)$ and define $n_{3}:=1 / n_{1}$ then we find

$$
m_{1}=m_{2}=m_{3}=m_{321}=i / n_{1}+n_{1} / i= \pm 2 \cos (\pi \mu) \text {. }
$$

Thus if this common value is not \pm 2 , the corresponding $\mathrm{SL}_{2}(\mathbb{C})$ matrices are regular semisimple and we have established the claim. However if this value is \pm 2 , it follows that $m=2$ contradicting the nondegeneracy assumption.

Such 'symmetric' $\mathrm{SL}_{2}(\mathbb{C})$ triples have been studied in this context by Hitchin (cf. [19, 20]), and that they arise from real reflection groups was known to Dubrovin-Mazzocco

(cf. [13] Remark 0.2). It is interesting to note that, from triples of generating reflections of the real three-dimensional tetrahedral, octahedral, and icosahedral reflection groups, one obtains in this way triples of generators of finite subgroups of $\mathrm{SL}_{2}(\mathbb{C})$. However, rather bizarrely, the tetrahedral and octahedral groups are swapped in the process: the tetrahedral reflection group maps to the binary octahedral subgroup of $\mathrm{SL}_{2}(\mathbb{C})$ and viceversa; the octahedral reflection group maps to the binary tetrahedral subgroup of $\mathrm{SL}_{2}(\mathbb{C})$. The three inequivalent triples of generating reflections of the icosahedral reflection group do all map to triples of generators of the binary icosahedral group though.

\section{ISOMONODROMIC DEFORMATIONS}

The main aim of this section is to see how the $\mathbb{C}^{*}$ action of (23) on the invariants of the pseudo-reflection data arises, and, on the other side of the Riemann-Hilbert correspondence, to describe the corresponding $\mathbb{C}$ action on the rank three Fuchsian systems.

This is the key ingredient needed to motivate the map $\varphi$ of (17), as described after Lemma 13 above. As a corollary we will see why $\varphi$ is $\mathcal{B}_{3}$-equivariant.

We will work in a somewhat more general context in this section than the rest of the paper; the reader interested mainly in the construction of algebraic solutions to PVI could skip straight to the next section.

Apart from the desire to explain how the procedure of section 2 was found, the motivation for this section is to enable us to see (in section (7) how one may work back from an explicit solution to PVI to an explicit rank three system of differential equations. This will give a mechanism for constructing new non-rigid systems of differential equations with finite monodromy group. (Except for this the proofs given in the other sections are independent of the results of this section.)

Let us begin with some generalities on isomonodromic deformations of Fuchsian systems and the Schlesinger equations. Let $V=\mathbb{C}^{n}$ and suppose we have matrices $B_{1}, \ldots, B_{m-1} \in$ $\operatorname{End}(V)$ and distinct points $a_{1}, \ldots, a_{m-1} \in \mathbb{C}$. Then consider the following meromorphic 
connection on the trivial rank $n$ holomorphic vector bundle over the Riemann sphere:

$$
\nabla:=d-\left(B_{1} \frac{d z}{z-a_{1}}+\cdots+B_{m-1} \frac{d z}{z-a_{m-1}}\right) .
$$

This has a simple pole at each $a_{i}$ and at infinity. Write

$$
B_{m}=B_{\infty}:=-\left(B_{1}+\cdots+B_{m-1}\right)
$$

for the residue matrix at infinity. Thus, on removing disjoint open discs $D_{1}, \ldots, D_{m}$ from around the poles and restricting $\nabla$ to the $m$-holed sphere

$$
S:=\mathbb{P}^{1} \backslash\left(D_{1} \cup \cdots \cup D_{m}\right),
$$

we obtain a (nonsingular) holomorphic connection. In particular it is flat and so, taking its monodromy, a representation of the fundamental group of the $m$-holed sphere is obtained. This procedure defines a holomorphic map, which we will call the monodromy map or Riemann-Hilbert map, from the set of such connection coefficients to the set of complex fundamental group representations:

$$
\left\{\left(B_{1}, \ldots, B_{m}\right) \mid \sum B_{i}=0\right\} \stackrel{\mathrm{RH}}{\longrightarrow}\left\{\left(M_{1}, \ldots, M_{m}\right) \mid M_{m} \cdots M_{1}=1\right\}
$$

where appropriate loops generating the fundamental group of $S$ have been chosen and the matrix $M_{i} \in G:=\mathrm{GL}_{n}(\mathbb{C})$ is the automorphism obtained by parallel translating a basis of solutions around the $i$ th loop.

The Schlesinger equations are the equations for isomonodromic deformations of the connection (25). Suppose we move the pole positions $a_{1}, \ldots, a_{m-1}$. Then we wish to vary the coefficients $B_{i}$, as functions of the pole positions, such that the monodromy data $\left(M_{1}, \ldots, M_{m}\right)$ only changes by diagonal conjugation by $G$. This is the case if the $B_{i}$ vary according to Schlesinger's equations:

$$
\frac{\partial B_{i}}{\partial a_{j}}=\frac{\left[B_{i}, B_{j}\right]}{a_{i}-a_{j}} \quad \text { if } i \neq j, \quad \text { and } \quad \frac{\partial B_{i}}{\partial a_{i}}=-\sum_{j \neq i, m} \frac{\left[B_{i}, B_{j}\right]}{a_{i}-a_{j}}
$$

where $i=1, \ldots, m-1$. Observe that these equations imply that the residue at infinity $B_{m}$ is held constant. Also note that the Schlesinger equations are equivalent to the flatness of the connection

$$
d-\left(B_{1} \frac{d z-d a_{1}}{z-a_{1}}+\cdots+B_{m-1} \frac{d z-d a_{m-1}}{z-a_{m-1}}\right) .
$$

In terms of differential forms Schlesinger's equations may be rewritten as

$$
d B_{i}=-\sum_{j \neq i, m}\left[B_{i}, B_{j}\right] d_{i j}
$$

where $d$ is the exterior derivative on $\left\{a_{i}\right\}$ and $d_{i j}:=d \log \left(a_{i}-a_{j}\right)=\left(d a_{i}-d a_{j}\right) /\left(a_{i}-a_{j}\right)$. In turn it will be convenient to rewrite this as

$$
d B_{i}=\left[L_{i}, B_{i}\right] \quad \text { where } \quad L_{i}:=\sum_{j \neq i, m} B_{j} d_{i j} .
$$

Note that if we have a local solution of Schlesinger's equations and we construct the $L_{i}$ from the formula (29) then firstly we have that $\nabla_{i}:=d-L_{i}$ is a flat connection and secondly that $B_{i}$ is a horizontal section of $\nabla_{i}$ (in the adjoint representation). 
Now let us specialise to the case where the dimension $n$ equals the number $m-1$ of finite singularities, and where each of the finite residues $B_{1}, \ldots, B_{n}$ is a rank one matrix. Thus

$$
B_{i}=f_{i} \otimes \beta_{i} \quad \text { for some } \quad f_{i} \in V, \beta_{i} \in V^{*} .
$$

Then we may lift the Schlesinger equations from the space of residues $B_{i}$ to the space of $f_{i}$ 's and $\beta_{i}$ 's. Namely, suppose we have a local solution of the Schlesinger equations on some polydisc $D$. Then we can write $B_{i}=f_{i} \otimes \beta_{i}$ for $i=1, \ldots, n$ at some base-point and evolve $f_{i}, \beta_{i}$ over $D$, as solutions to the equations:

$$
d f_{i}=L_{i} f_{i} \quad d \beta_{i}=-\beta_{i} L_{i}
$$

where the $L_{i}$ are defined in terms of the given $B_{j}$ solving the Schlesinger equations. Then one finds immediately that the $f_{i} \otimes \beta_{i}$ solve (29), and so $f_{i} \otimes \beta_{i}=B_{i}$ throughout $D$ (since they agree at the basepoint and solve Schlesinger's equations). Alternatively one can view (30) as a coupled system of nonlinear equations for $\left\{f_{i}, \beta_{i}\right\}$, by defining $L_{i}$ in terms of the $B_{j}:=f_{j} \otimes \beta_{j}$ as in (29). We will refer to these as the lifted equations (they were introduced in [25] and further studied in [16]). The above considerations show:

Proposition 15. Any solution of the Schlesinger equations (26) may be lifted to a solution of the lifted equations (30) by only solving linear equations. Conversely any solution of the lifted equations projects to a solution of (26) by setting $B_{i}=f_{i} \otimes \beta_{i}$.

Now we wish to define an action of $\mathbb{C}$ which will be the additive analogue of the $\mathbb{C}^{*}$ action of (23).

Suppose we have a local solution $\left\{B_{1}(\mathbf{a}), \ldots, B_{n}(\mathbf{a})\right\}$ of Schlesinger's equations on some polydisc $D$, where $\mathbf{a}=\left(a_{1}, \ldots, a_{n}\right)$, such that the images of the $B_{i}$ are linearly independent (i.e. for any choice of $f_{i}, \beta_{i}$ such that $B_{i}=f_{i} \otimes \beta_{i}$, the $f_{i}$ make up a basis of $V$ ). Then we can define the following action of the complex numbers on the set of such solutions:

Proposition 16. For any complex number $\lambda \in \mathbb{C}$ the matrices

$$
\widetilde{B}_{i}:=B_{i}+\lambda f_{i} \otimes \widehat{f}_{i}
$$

constitute another solution to Schlesinger's equations on $D$, where $\widehat{f}_{1}, \ldots, \widehat{f}_{n} \in V^{*}$ are the dual basis defined by $\widehat{f}_{i}\left(f_{j}\right)=\delta_{i j}$.

Proof. First note that this is well-defined since the projectors $f_{i} \otimes \widehat{f}_{i}$ are independent of the choice of $f_{i}$ 's. Then lift the $B_{i}$ arbitrarily to a solution $\left\{f_{i}(\mathbf{a}), \beta_{i}(\mathbf{a})\right\}$ of (30) over $D$. Straightforward computations then give that $\widehat{f}_{i}$ satisfies $d \widehat{f}_{i}=-\sum_{j \neq i} \beta_{i}\left(f_{j}\right) \widehat{f}_{j} d_{i j}$ and using this one easily confirms $d \widetilde{B}_{i}=\left[\widetilde{L}_{i}, \widetilde{B}_{i}\right]$ where $\widetilde{L}_{i}=L_{i}+\lambda \sum_{j \neq i} f_{j} \otimes \widehat{f}_{j} d_{i j}$.

One may arrive at this action as follows. Given a local solution $\left\{f_{i}(\mathbf{a}), \beta_{i}(\mathbf{a})\right\}$ of the lifted equations one may check that the matrix $B \in \operatorname{End}(V)$ defined by $(B)_{i j}=\beta_{i}\left(f_{j}\right)$ satisfies the nonlinear differential equation

$$
d B=\left[B, \operatorname{ad}_{A_{0}}^{-1}\left(\left[d A_{0}, B\right]\right)\right]
$$

where $A_{0}:=\operatorname{diag}\left(a_{1}, \ldots, a_{n}\right) . \quad\left(\right.$ Note that $\operatorname{ad}_{A_{0}}: \operatorname{End}(V) \rightarrow \operatorname{End}(V)$ is invertible when restricted to the matrices with zero diagonal part and that $\left[d A_{0}, B\right]$ has zero diagonal part.) This is the 'dual' equation to the Schlesinger equations in the present context (in 
the sense of Harnad [16]) and arises as the equation for isomonodromic deformations of the irregular connection

$$
d-\left(\frac{A_{0}}{w^{2}}+\frac{B}{w}\right) d w,
$$

which, after an appropriate coordinate change, appears as the (twisted) Fourier-Laplace transform of the original Fuchsian system (cf. [1] and references therein). Equation (31) appears in the theory of Frobenius manifolds [1] for skew-symmetric $B$ and is related to quantum Weyl groups [5]. Note that equation (31) is equivalent to the Schlesinger equations in that its solutions may also be lifted to solutions of (30) by only solving the linear equations

$$
d f_{i}=\sum_{j \neq i}(B)_{j i} f_{j} d_{i j} \quad d \beta_{i}=-\sum_{j \neq i}(B)_{i j} \beta_{j} d_{i j}
$$

where $B(\mathbf{a})$ solves (31).

Now from the form of (31) it is transparent that replacing $B$ by $B+\lambda$ maps solutions to solutions (where $\lambda \in \mathbb{C}$ is constant). (Observe this corresponds to tensoring the irregular connection (31) by the meromorphic connection $d-\lambda \frac{d w}{w}$ on the trivial line bundle.) If $B$ is translated in this way, then (provided the $f_{i}$ are a basis) we can see how to change the corresponding Schlesinger solutions as follows. First note:

Lemma 17. Suppose $f_{1}, \ldots, f_{n} \in V$ is an arbitrary basis, $\beta_{i} \in V^{*}$ is arbitrary, $B_{i}=$ $f_{i} \otimes \beta_{i} \in \operatorname{End}(V)$ and $(B)_{i j}=\beta_{i}\left(f_{j}\right)$. Then

$$
\left(B_{1}, \ldots, B_{n}\right) \text { is conjugate to }\left(E_{1} B, \ldots, E_{n} B\right)
$$

where $E_{i} \in \operatorname{End}(V)$ has $(i, i)$ entry 1 and is otherwise zero.

Proof. Define $g \in \mathrm{GL}(V)$ to have $i$ th column $f_{i}$. Then observe that $g^{-1} B_{i} g=E_{i} B$.

Thus replacing $B$ by $B+\lambda$ changes $B_{i}=g E_{i} B g^{-1}$ to $B_{i}+\lambda g E_{i} g^{-1}=B_{i}+\lambda f_{i} \otimes \widehat{f_{i}}$ and so we deduce the action of Proposition [16.

Now the basic idea to reduce the rank of the systems by one is to choose $\lambda$ to be an eigenvalue of $B_{\infty}$. Then the residue at infinity $\widetilde{B}_{\infty}$ of the resulting system has a nontrivial kernel. This is because Lemma 17 implies

$$
B_{\infty}=-\left(B_{1}+\cdots+B_{n}\right) \quad \text { is conjugate to } \quad-\left(E_{1} B+\cdots+E_{n} B\right)=-B .
$$

Thus translating $B$ by $\lambda$ implies that $\widetilde{B}_{\infty}$ is conjugate to $B_{\infty}-\lambda$, which will have a zero eigenvalue. Say $\widetilde{B}_{\infty} v=0$ for some vector $v$. Then the fact that the $f_{i}$ are a basis implies that $v$ is in the kernel of all the residues $\widetilde{B}_{i}$ - the resulting system is reducible and we can pass to the corresponding $(n-1) \times(n-1)$ quotient system.

The next step is to find the action on monodromy data corresponding to the $\mathbb{C}$ action above. Suppose $B_{j}=f_{j} \otimes \beta_{j}$ for $j=1, \ldots, n$ and each

$$
\lambda_{j}:=\operatorname{Tr}\left(B_{j}\right)=\beta_{j}\left(f_{j}\right)
$$

is not an integer. Then one knows that the monodromy matrix $M_{j}$ around $a_{j}$ is conjugate to $\exp \left(2 \pi i B_{j}\right)$ and so is a (diagonalisable) pseudo-reflection. We will write

$$
r_{j}=M_{j}=1+e_{j} \otimes \alpha_{j} \quad \text { where } e_{j} \in V, \alpha_{j} \in V^{*}
$$


for this pseudo-reflection. Clearly the non-identity eigenvalue of $r_{j}$ is $\exp \left(2 \pi i \lambda_{j}\right)$ so setting $t_{j}=\exp \left(\pi i \lambda_{j}\right)($ as in (19) $)$ implies $t_{j}^{2}=\operatorname{det}\left(r_{j}\right)$ agreeing with the definition (13).

From (33) we deduce that if $B$ is translated by $\lambda$ then the monodromy around a large positive loop is just scaled:

$$
\widetilde{r}_{n} \cdots \widetilde{r}_{2} \widetilde{r}_{1} \text { is conjugate to } r_{n} \cdots r_{2} r_{1} h^{2}
$$

where

$$
h:=\exp (\pi i \lambda) \in \mathbb{C}^{*},
$$

at least if $B_{\infty}$ is sufficiently generic (no distinct eigenvalues differing by integers). (Here $\widetilde{r}_{1}, \ldots, \widetilde{r}_{n}$ are the monodromy data of the connection obtained by replacing each $B_{i}$ by $\widetilde{B}_{i}$ in (25).)

In brief the additive action was determined by the fact that sum $\sum B_{i}$ was just translated by $\lambda$ (assuming the $f_{i}$ make up a basis, which is held fixed). We will see below that the multiplicative action (i.e. the action on monodromy data) is determined by the fact that the product $r_{n} \cdots r_{1}$ is just scaled by $h^{2}$ (assuming the $e_{i}$ make up a basis, which is held fixed).

First let us recall a basic algebraic fact about pseudo-reflections.

Suppose $e_{1}, \ldots, e_{n}$ are a basis of $V$ and $\alpha_{1}, \ldots, \alpha_{n} \in V^{*}$ are such that $r_{i}:=1+e_{i} \otimes \alpha_{i} \in$ $\mathrm{GL}(V)$, i.e. $1+\alpha_{i}\left(e_{i}\right) \neq 0$. Define two $n \times n$ matrices $t^{2}, u$ by

$$
t^{2}:=\operatorname{diag}\left(1+\alpha_{1}\left(e_{1}\right), \ldots, 1+\alpha_{n}\left(e_{n}\right)\right), \quad(u)_{i j}=\alpha_{i}\left(e_{j}\right) .
$$

(We do not need to choose a square root $t$ of $t^{2}$ at this stage, but it is convenient to keep the notation consistent with other sections of the paper.) Then let $u_{+}, u_{-}$be the two unipotent matrices determined by the equation

$$
t^{2} u_{+}-u_{-}=u
$$

where $u_{+}$is upper triangular with ones on the diagonal and $u_{-}$is lower triangular with ones on the diagonal.

Theorem 2 (Killing [26], Coxeter [9]). The matrix representing the product $r_{n} \cdots r_{1}$ (in the $e_{i}$ basis) is in the big-cell of $\mathrm{GL}_{n}(\mathbb{C})$, and so may be written uniquely as the product of a lower triangular, a diagonal and an upper triangular matrix. Moreover this factorisation is given explicitly by $u_{-}^{-1} t^{2} u_{+}$:

$$
r_{n} \cdots r_{2} r_{1}=u_{-}^{-1} t^{2} u_{+}
$$

Remark 18. The history of this result is discussed by Coleman [8] (cf. Corollary 3.4). Coxeter proves this for genuine reflections - i.e. coming from a symmetric bilinear form in [9]. The starting point of this paper was the simple observation that Coxeter's argument may be extended to the pseudo-reflection case. Dubrovin had used Coxeter's version in relation to Frobenius manifolds (cf. [12]) and the author was interested in extending Dubrovin's picture to the general case (cf. 3]). Despite asking various complex reflection group experts the author only found Coleman's paper (and hence the link to Killing) since [8] is in the same volume as a well-known paper of Beukers-Heckman.

It is worth clarifying the fact that generically the matrix $u$ determines $\left(r_{1}, \ldots, r_{n}\right)$ up to conjugacy: 
Lemma 19. If $\operatorname{det}(u) \neq 0$ then there is a matrix $g \in \mathrm{GL}(V)$ such that, for $i=1, \ldots, n$ we have

$$
r_{i}=g\left(1+e_{i}^{o} \otimes \gamma_{i}\right) g^{-1}
$$

where $\gamma_{i} \in V^{*}$ is the ith row of the matrix $u$ and $e_{i}^{o}$ is the standard basis of $V$.

Proof. By definition of $u$, if $\operatorname{det}(u) \neq 0$ then the $e_{i}$ are a basis of $V$. Then the result follows since we know the action on a basis: $r_{i}\left(e_{j}\right)=e_{j}+u_{i j} e_{i}$.

Note that if we define $u_{ \pm}, t^{2}$ by the equation $u=t^{2} u_{+}-u_{-}$then the condition that $\operatorname{det}(u) \neq 0$ is equivalent to saying 1 is not an eigenvalue of $u_{-}^{-1} t^{2} u_{+}$, since $\operatorname{det}(u)=$ $\operatorname{det}\left(t^{2} u_{+}-u_{-}\right)=\operatorname{det}\left(u_{-}^{-1} t^{2} u_{+}-1\right)$.

Thus generically the $n$-tuple $\left(r_{1}, \ldots, r_{n}\right)$ is determined up to overall conjugacy by the matrix $u$, and in turn by the product $r_{n} \cdots r_{1}$, by Theorem 2 . From (34) the obvious guess is therefore that that $\widetilde{r}_{n} \cdots \widetilde{r}_{1}=u_{-}^{-1} t^{2} u_{+} h^{2}=u_{-}^{-1} h^{2} t^{2} u_{+}$so that $\widetilde{\alpha}_{i}\left(\widetilde{e}_{j}\right)=\left(h^{2} t^{2} u_{+}-u_{-}\right)_{i j}$, which should determine $\left(\widetilde{r}_{1}, \ldots, \widetilde{r}_{n}\right)$ up to overall conjugacy. The following theorem says that this is indeed the case, at least generically. Suppose each $\lambda_{i}$ and each eigenvalue of $\sum B_{i}$ is not an integer (and that the same holds after translation by $\lambda$ ). Then we have:

Theorem 3 (Balser-Jurkat-Lutz [1]). Let $\widetilde{u}$ be the matrix

$$
\widetilde{u}=h^{2} t^{2} u_{+}-u_{-}
$$

where $u_{ \pm}, t^{2}, h$ are as defined above. Then there is a basis $\widetilde{e}_{i}$ of $V=\mathbb{C}^{n}$ and $\widetilde{\alpha}_{1}, \ldots \widetilde{\alpha}_{n} \in V^{*}$ such that $\widetilde{r}_{i}=1+\widetilde{e}_{i} \otimes \widetilde{\alpha}_{i}$ and $(\widetilde{u})_{i j}=\widetilde{\alpha}_{i}\left(\widetilde{e}_{j}\right)$ for all $i, j$.

Remark 20. This is not written down in precisely this way in [1] so we will describe how to extract it in Appendix A. The key point is that the matrices $u_{ \pm}$are essentially the Stokes matrices of the irregular connection (32) and are easily seen to be preserved under the scalar shift. Then one computes bases of solutions of the Fuchsian connection as Laplace transforms of standard bases of solutions of (32) and this enables the Stokes matrices to be related to the pseudo-reflection data $u$ as in equation (35). The observation that this implies the Fuchsian monodromy data and the Stokes data are then related by the beautiful equation (36) in Theorem 2 does not seem to appear in [1. In summary we see that equation (36) is the manifestation of the Fourier-Laplace transformation on monodromy data, relating the monodromy data of the Fuchsian connection to the monodromy/Stokes data of the corresponding irregular connection.

In other words: in general the matrix $u$ determines $\left(r_{1}, \ldots, r_{n}\right)$ up to overall conjugation and Theorem 3 explains how the matrix $u$ varies: the lower triangular part is fixed, the upper triangular part is scaled by $h^{2}$, and the diagonal part $t^{2}-1$ becomes $h^{2} t^{2}-1$. Let us make this more explicit in the $n=3$ case. We start with a connection

$$
d-\sum_{1}^{3} \frac{B_{i}}{z-a_{i}} d z
$$

where, up to overall conjugation:

$$
B_{1}=\left(\begin{array}{ccc}
\lambda_{1} & b_{12} & b_{13} \\
0 & 0 & 0 \\
0 & 0 & 0
\end{array}\right), \quad B_{2}=\left(\begin{array}{ccc}
0 & 0 & 0 \\
b_{21} & \lambda_{3} & b_{23} \\
0 & 0 & 0
\end{array}\right), \quad B_{3}=\left(\begin{array}{ccc}
0 & 0 & 0 \\
0 & 0 & 0 \\
b_{31} & b_{32} & \lambda_{3}
\end{array}\right)
$$


for some numbers $b_{i j}$ with $i \neq j$. Then we take the monodromy data of this and obtain pseudo-reflections $r_{1}, r_{2}, r_{3}$ which, up to overall conjugation, are of the form

$$
r_{1}=\left(\begin{array}{ccc}
t_{1}^{2} & u_{12} & u_{13} \\
0 & 1 & 0 \\
0 & 0 & 1
\end{array}\right), \quad r_{2}=\left(\begin{array}{ccc}
1 & 0 & 0 \\
u_{21} & t_{2}^{2} & u_{23} \\
0 & 0 & 1
\end{array}\right), \quad r_{3}=\left(\begin{array}{ccc}
1 & 0 & 0 \\
0 & 1 & 0 \\
u_{31} & u_{32} & t_{3}^{2}
\end{array}\right)
$$

where $t_{j}=\exp \left(\pi i \lambda_{j}\right)$. Then we replace $\lambda_{i}$ by $\lambda_{i}+\lambda$ in (37) for each $i$, and Theorem 3 says that the monodromy of the resulting connection is conjugate to

$$
\widetilde{r}_{1}=\left(\begin{array}{ccc}
h^{2} t_{1}^{2} & h^{2} u_{12} & h^{2} u_{13} \\
0 & 1 & 0 \\
0 & 0 & 1
\end{array}\right), \widetilde{r}_{2}=\left(\begin{array}{ccc}
1 & 0 & 0 \\
u_{21} & h^{2} t_{2}^{2} & h^{2} u_{23} \\
0 & 0 & 1
\end{array}\right), \widetilde{r}_{3}=\left(\begin{array}{ccc}
1 & 0 & 0 \\
0 & 1 & 0 \\
u_{31} & u_{32} & h^{2} t_{3}^{2}
\end{array}\right)
$$

where $h:=\exp (\pi i \lambda)$.

Taking the various traces yields the fact that the invariant functions of the monodromy matrices are related as:

$$
\widetilde{t}_{i}^{2}=h^{2} t_{i}^{2}, \quad \widetilde{t}_{i j}=h^{2} t_{i j}, \quad \widetilde{t}_{321}=h^{2} t_{321}, \quad \widetilde{t}_{321}^{\prime}=h^{4} t_{321}^{\prime} .
$$

These equations hold for any $\lambda$ since the invariants are analytic functions of the coefficients $B_{i}$ and so vary holomorphically with the parameter $\lambda$. This motivates the definition of the $\mathbb{C}^{*}$ action in (23), and in turn this yields the definition of the map $\varphi$ as explained just after Lemma 13, by taking the projection to $\mathrm{SL}_{2}(\mathbb{C})$ of the rank two part of the semisimplification of (38) when $\lambda=-\mu_{1}$. (Note that in our conventions the $\mu_{i}$ are the eigenvalues of $\sum B_{i}=-B_{\infty}$.)

\section{Braid group actions.}

Let us check that the $\mathbb{C}^{*}$ action commutes with the braid group action on the level of the matrices $u$. (One suspects this is the case since the braid group actions are obtained by integrating the isomonodromy equations, and we have seen in Proposition [16 that the corresponding $\mathbb{C}$ action commutes with the Schlesinger flows.)

The standard braid group action of the $n$-string braid group $\mathcal{B}_{n}$ on $n$-tuples of pseudoreflections may be given by generators $\gamma_{1}, \ldots, \gamma_{n-1}$ with $\gamma_{i}$ acting as

$$
\gamma_{i}\left(r_{n}, \ldots, r_{1}\right)=\left(\ldots, r_{i+2}, r_{i}, r_{i}^{-1} r_{i+1} r_{i}, r_{i-1}, \ldots\right)
$$

only affecting $r_{i}, r_{i+1}$ and preserving the product $r_{n} \cdots r_{1}$. (For $n=3$ we previously labelled the generators differently: $\beta_{1}=\gamma_{2}, \beta_{2}=\gamma_{1}$.) Now suppose we write $r_{i}=1+e_{i} \otimes \alpha_{i}$ with $e_{i} \in V, \alpha_{i} \in V^{*}$, where $V=\mathbb{C}^{n}$. Let us restrict to the case where the $r_{i}$ are linearly independent (in the sense that any such $e_{i}$ form a basis of $V$ ). Then it is easy to lift the above $\mathcal{B}_{n}$ action to an action on the $2 n^{2}$-dimensional space

$$
W:=\left\{\left(e_{n}, \ldots, e_{1}, \alpha_{n}, \ldots \alpha_{1}\right) \mid\left\{e_{i}\right\} \text { a basis of } V, \alpha_{i} \in V^{*}, \alpha_{i}\left(e_{i}\right) \neq-1\right\}
$$

by letting $\gamma_{i}$ fix all $e_{j}, \alpha_{j}$ except for $j=i, i+1$ :

$$
\gamma_{i}\left(\ldots, e_{i+1}, e_{i}, \ldots, \alpha_{i+1}, \alpha_{i}, \ldots\right)=\left(\ldots, e_{i}, r_{i}^{-1} e_{i+1}, \ldots, \alpha_{i}, \alpha_{i+1} \circ r_{i}, \ldots\right)
$$

where $r_{i}:=1+e_{i} \otimes \alpha_{i} \in \mathrm{GL}(V)$. (We think of $W$ as the multiplicative analogue of the space on which the lifted equations (30) were defined.) It is simple to check this action is well-defined on $W$. 
Now we may project this lifted $\mathcal{B}_{n}$-action to the space of the matrices $u$. Recall the $n \times n$ matrix $u$ was defined by setting $u_{i j}=\alpha_{i}\left(e_{j}\right)$. By a straightforward computation we find that, if we set $u^{\prime}=\gamma_{i}(u)$ then $u_{j k}^{\prime}=u_{j k}$ unless one of $j$ or $k$ equals $i$ or $i+1$, and

$$
\begin{aligned}
u_{i i}^{\prime}=u_{i+1 i+1}, & u_{i+1 i+1}^{\prime}=u_{i i} \\
u_{i i+1}^{\prime}=t_{i}^{2} u_{i+1 i}, & u_{i+1 i}^{\prime}=u_{i i+1} / t_{i}^{2} \\
u_{i j}^{\prime}=u_{i+1 j}+u_{i+1 i} u_{i j}, \quad & u_{j i}^{\prime}=u_{j i+1}-u_{j i} u_{i i+1} / t_{i}^{2} \\
u_{i+1 j}^{\prime}=u_{i j}, & u_{j i+1}^{\prime}=u_{j i}
\end{aligned}
$$

for any $j \notin\{i, i+1\}$ where $t_{i}^{2}:=1+u_{i i}$.

In turn $u$ contains precisely the same data as the matrix

$$
u_{-}^{-1} t^{2} u_{+} \in G^{0} \subset \mathrm{GL}_{n}(\mathbb{C})
$$

where $u_{ \pm}, t^{2}$ are determined by the equation $u=t^{2} u_{+}-u_{-}$, and $G^{0}$ denotes the big-cell, consisting of the invertible matrices that may be factorised as the product of a lower triangular and an upper triangular matrix. Thus the $\mathcal{B}_{n}$-action on $\{u\}$ is equivalent to a $\mathcal{B}_{n}$-action on $G^{0}$. Let us describe this. First let $P_{i} \in \mathrm{GL}_{n}(\mathbb{C})$ denote the permutation matrix corresponding to the permutation swapping $i$ and $i+1$. Thus $P_{i}$ equals the identity matrix except in the $2 \times 2$ block in the $i, i+1$ position on the diagonal, where it equals $\left(\begin{array}{ll}0 & 1 \\ 1 & 0\end{array}\right)$. Also for any unipotent upper triangular matrix $u_{+}$, let $\xi_{i}\left(u_{+}\right)$denote the matrix which equals the identity matrix except in the $2 \times 2$ block in the $i, i+1$ position on the diagonal, where it equals that of $u_{+}$, namely

$$
\left(\begin{array}{cc}
1 & \left(u_{+}\right)_{i i+1} \\
0 & 1
\end{array}\right)
$$

(This map $\xi_{i}$ defines a homomorphism from $U_{+}$to the root group of $\mathrm{GL}_{n}(\mathbb{C})$ corresponding to the $i$ th simple root-cf. e.g. (3.10) [5].)

Proposition 21. The induced $\mathcal{B}_{n}$-action on $G^{0}$ is given by the formula

$$
\gamma_{i}(a)=P_{i} \xi_{i}\left(u_{+}\right) a \xi_{i}\left(u_{+}\right)^{-1} P_{i} .
$$

where $a=u_{-}^{-1} t^{2} u_{+} \in G^{0}$.

Proof. Lifting back up to $W$, write $r_{j}=1+e_{j} \otimes \alpha_{j}$ and denote

$$
\left(\ldots, e_{i+1}^{\prime}, e_{i}^{\prime}, \ldots, \alpha_{i+1}^{\prime}, \alpha_{i}^{\prime}, \ldots\right)=\gamma_{i}\left(\ldots, e_{i+1}, e_{i}, \ldots, \alpha_{i+1}, \alpha_{i}, \ldots\right) .
$$

Note that the product $R:=r_{n} \cdots r_{1} \in \mathrm{GL}(V)$ is fixed by the $\mathcal{B}_{n}$ action. By Theorem 2 the matrix for $R$ in the $e_{j}$ basis of $V$ is $a=u_{-}^{-1} t^{2} u_{+}$, and in the $e_{j}^{\prime}$ basis the matrix for $R$ is $\gamma_{i}(a)$. Thus $\gamma_{i}(a)=S^{-1} a S$ where $S$ is the matrix for the change of basis from $\left\{e_{j}\right\}$ to $\left\{e_{j}^{\prime}\right\}$. From the formula for the action on the $e_{j}, S$ equals the identity matrix except in the $2 \times 2$ block in the $i, i+1$ position on the diagonal, where it equals

$$
\left(\begin{array}{cc}
-u_{i i+1} / t_{i}^{2} & 1 \\
1 & 0
\end{array}\right)=\left(\begin{array}{cc}
1 & u_{i i+1} / t_{i}^{2} \\
0 & 1
\end{array}\right)^{-1}\left(\begin{array}{ll}
0 & 1 \\
1 & 0
\end{array}\right) .
$$

Finally from the equation $u=t^{2} u_{+}-u_{-}$we see $u_{i i+1} / t_{i}^{2}=\left(u_{+}\right)_{i i+1}$ so $S=\xi_{i}\left(u_{+}\right)^{-1} P_{i}$. 
Remark 22. This $\mathcal{B}_{n}$ action on the big-cell also appears as the classical limit of the socalled quantum Weyl group actions (cf. [10] and [5] Remark 3.8), provided we use the permutation matrices rather than Tits' extended Weyl group. Thus we have shown, for $\mathrm{GL}_{n}(\mathbb{C})$, how the classical action of the quantum Weyl group is related to the standard action of $\mathcal{B}_{n}$ on $n$-tuples of pseudo-reflections. Presumably this is related to Toledano Laredo's proof 32 , for $\mathrm{GL}_{n}(\mathbb{C})$ of the Kohno-Drinfeld theorem for quantum Weyl groups.

Corollary 23. The $\mathbb{C}^{*}$ action on $\{u\}$ commutes with the $\mathcal{B}_{n}$-action defined above.

Proof. On passing to $G^{0}$, we recall that the $\mathbb{C}^{*}$ action just scales $t^{2}$ and leaves both $u_{ \pm}$fixed. However $t^{2}$ does not appear in the formula of Proposition 21 for the $\mathcal{B}_{n}$ action.

One can now see directly why the map $\varphi$ will be $\mathcal{B}_{3}$ equivariant. Upon using the $\mathbb{C}^{*}$ action to make 1 an eigenvalue of $r_{3} r_{2} r_{1}$ we know that the $r_{i}$ are all block triangular in some basis. Then we just note the obvious fact that the braid group action (14) on the pseudo-reflections restricts to the action (8) in the $2 \times 2$ block on the diagonal.

\section{JIMBO'S LEADING TERM FORMULA}

So far we have described how to find some $\mathrm{SL}_{2}(\mathbb{C})$ triples living in finite orbits of the braid group, and read off some properties of the corresponding solution to Painlevé VI (in particular we saw that the set of branches of the solution correspond to the orbit under the pure braid group of the conjugacy classes of such triples). In this section and the next we will describe a method to find the corresponding solution explicitly. This method is quite general and should work with any sufficiently generic $\mathrm{SL}_{2}$ triple in a finite braid group orbit - in particular it is not a priori restricted to any one-parameter family of Painlevé VI equations. (One just needs to check conditions b),c),d) below for each branch of the solution.)

The crucial step is the following formula:

Theorem 4. (M. Jimbo [23]) Suppose we have four matrices $M_{j} \in \mathrm{SL}_{2}(\mathbb{C}), j=0, t, 1, \infty$ satisfying

a) $M_{\infty} M_{1} M_{t} M_{0}=1$,

b) $M_{j}$ has eigenvalues $\left\{\exp \left( \pm \pi i \theta_{j}\right)\right\}$ with $\theta_{j} \notin \mathbb{Z}$,

c) $\operatorname{Tr}\left(M_{0} M_{t}\right)=2 \cos (\pi \sigma)$ for some nonzero $\sigma \in \mathbb{C}$ with $0 \leq \operatorname{Re}(\sigma)<1$,

d) None of the eight numbers

$$
\theta_{0} \pm \theta_{t} \pm \sigma, \quad \theta_{0} \pm \theta_{t} \mp \sigma, \quad \theta_{\infty} \pm \theta_{1} \pm \sigma, \quad \theta_{\infty} \pm \theta_{1} \mp \sigma
$$

is an even integer.

Then the leading term in the asymptotic expansion at zero of the corresponding Painlevé $V I$ solution $y(t)$ on the branch corresponding to $\left[\left(M_{0}, M_{t}, M_{1}\right)\right]$ is

$$
\frac{\left(\theta_{0}+\theta_{t}+\sigma\right)\left(-\theta_{0}+\theta_{t}+\sigma\right)\left(\theta_{\infty}+\theta_{1}+\sigma\right)}{4 \sigma^{2}\left(\theta_{\infty}+\theta_{1}-\sigma\right) \widehat{s}} t^{1-\sigma}
$$

where

$$
\begin{gathered}
\widehat{s}=c \times s, \quad s=\frac{a+b}{d} \\
a=e^{\pi i \sigma}\left(i \sin (\pi \sigma) \cos \left(\pi \sigma_{1 t}\right)-\cos \left(\pi \theta_{t}\right) \cos \left(\pi \theta_{\infty}\right)-\cos \left(\pi \theta_{0}\right) \cos \left(\pi \theta_{1}\right)\right)
\end{gathered}
$$




$$
\begin{gathered}
b=i \sin (\pi \sigma) \cos \left(\pi \sigma_{01}\right)+\cos \left(\pi \theta_{t}\right) \cos \left(\pi \theta_{1}\right)+\cos \left(\pi \theta_{\infty}\right) \cos \left(\pi \theta_{0}\right) \\
d=4 \sin \left(\frac{\pi}{2}\left(\theta_{0}+\theta_{t}-\sigma\right)\right) \sin \left(\frac{\pi}{2}\left(\theta_{0}-\theta_{t}+\sigma\right)\right) \sin \left(\frac{\pi}{2}\left(\theta_{\infty}+\theta_{1}-\sigma\right)\right) \sin \left(\frac{\pi}{2}\left(\theta_{\infty}-\theta_{1}+\sigma\right)\right) \\
c=\frac{(\Gamma(1-\sigma))^{2} \widehat{\Gamma}\left(\theta_{0}+\theta_{t}+\sigma\right) \widehat{\Gamma}\left(-\theta_{0}+\theta_{t}+\sigma\right) \widehat{\Gamma}\left(\theta_{\infty}+\theta_{1}+\sigma\right) \widehat{\Gamma}\left(-\theta_{\infty}+\theta_{1}+\sigma\right)}{(\Gamma(1+\sigma))^{2} \widehat{\Gamma}\left(\theta_{0}+\theta_{t}-\sigma\right) \widehat{\Gamma}\left(-\theta_{0}+\theta_{t}-\sigma\right) \widehat{\Gamma}\left(\theta_{\infty}+\theta_{1}-\sigma\right) \widehat{\Gamma}\left(-\theta_{\infty}+\theta_{1}-\sigma\right)}
\end{gathered}
$$

where $\widehat{\Gamma}(x):=\Gamma\left(\frac{1}{2} x+1\right)$ (with $\Gamma$ being the usual gamma function) and where $\sigma_{j k} \in \mathbb{C}$ (for $j, k \in\{0, t, 1\})$ is determined by $\operatorname{Tr}\left(M_{j} M_{k}\right)=2 \cos \left(\pi \sigma_{j k}\right), 0 \leq \operatorname{Re}\left(\sigma_{j k}\right) \leq 1$, so $\sigma=\sigma_{0 t}$.

Remark 24. The formula (40) is computed directly from the formula [23] (2.15) for the asymptotics as $t \rightarrow 0$ for the coefficients of the isomonodromic family of rank two systems. The formulae for $\widehat{s}$ and $s$ are as in [23] except for a sign difference in $s$. Since this formula is crucial for us and since $s$ is not derived in [23] we will give a derivation in the appendix.

Remark 25. D. Guzzetti repeated Jimbo's computations in [15] Section 8.3 and Appendix, but did not reduce the formula to as short a form; see [15] (A.6) and (A.30) (but note (A.30) is not quite correct but is easily corrected by examining (A.28) and (A.29)). However we can state that the corrected version of Jimbo's formula agrees numerically with the corrected version of Guzzetti's (at least for the values of the parameters used in this paper, and for those of several hundred randomly chosen $\mathrm{SL}_{2}(\mathbb{C})$ triples). It is puzzling that Guzzetti does not state that his formula does not agree with Jimbo's. ${ }^{4}$

Remark 26. To agree with Jimbo's notation we are thus relabelling the triples $\left(M_{1}, M_{2}, M_{3}\right)$ as $\left(M_{0}, M_{t}, M_{1}\right)$ as well as the $\theta$ parameters $\left(\theta_{1}, \theta_{2}, \theta_{3}, \theta_{4}\right) \mapsto\left(\theta_{0}, \theta_{t}, \theta_{1}, \theta_{\infty}\right)$. To keep track of this it is perhaps simplest to bear in mind the corresponding monodromy relations

$$
M_{4} M_{3} M_{2} M_{1}=1 \quad \text { and } \quad M_{\infty} M_{1} M_{t} M_{0}=1 .
$$

\section{The Klein SOlution}

For the Klein solution $\sigma$ is either $1 / 2$ or $1 / 3$ depending on the branch. If the solution is to be algebraic then Jimbo's formula will give the leading term in the Puiseux expansion at 0 of each branch of the solution. Thus we find the leading term on the $j$ th branch is of the form $C_{j} t^{1-\sigma_{j}}$ where

$$
C_{j}=\frac{57}{28 \widehat{s}_{j}}
$$

on the four branches with $\sigma=1 / 2(j=0,1,2,3)$ and

$$
C_{j}=\frac{475}{308 \widehat{s}_{j}}
$$

on the other three branches, having $\sigma=1 / 3(j=4,5,6)$. Now we would like to evaluate these precisely on each branch and identify them as algebraic numbers. A simple numerical inspection shows that $C_{0}$ has argument $\pi / 4, C_{6}$ is real and negative,

$$
C_{1}=-i C_{0}, \quad C_{2}=i C_{0}, \quad C_{3}=-C_{0}
$$

\footnotetext{
${ }^{4}$ Also there is some confusion as to the range of validity of Jimbo's work: namely the restriction $0 \leq$ $\operatorname{Re}(\sigma)<1$, is equivalent to $\operatorname{Tr}\left(M_{0} M_{t}\right) \notin \mathbb{R}_{\leq-2}$ rather than the much stronger condition $\left|\operatorname{Tr}\left(M_{0} M_{t}\right)\right| \leq 2$ and $\operatorname{Re} \operatorname{Tr}\left(M_{0} M_{t}\right) \neq-2$ appearing in [15] (1.30).
} 
and

$$
C_{4}=\exp (-2 \pi i / 3) C_{6}, \quad C_{5}=\exp (2 \pi i / 3) C_{6} .
$$

Thus we would hope that $C_{0}^{4}$ and $C_{6}^{3}$ are rational numbers. Using Maple we calculate the various $\widehat{s}_{j}$ 's numerically and then deduce:

$$
\begin{gathered}
C_{0}^{4}=-7 / 3^{4}, \quad \text { so that } \quad C_{0}=\frac{(1+i) 7^{1 / 4}}{3 \sqrt{2}} \\
C_{6}^{3}=-5^{3} / 14, \quad \text { so that } \quad C_{6}=\frac{-5}{14^{1 / 3}} .
\end{gathered}
$$

Thus we now know precisely the leading coefficient $C_{j}$ of the Puiseux expansion at 0 of each branch of the solution $y(t)$. By substituting back into the Painlevé VI equation these leading terms determine, algebraically, any desired term in the Puiseux expansion. If the solution is to be algebraic it should satisfy an equation of the form

$$
F(t, y(t))=0
$$

for some polynomial $F(t, y)$ in two variables. Since the solution has 7 branches $F$ should have degree 7 in $y$. Let us write $F$ in the form

$$
F=q(t) y^{7}+p_{6}(t) y^{6}+\cdots+p_{1}(t) y+p_{0}(t)
$$

for polynomials $p_{i}, q$ in $t$ and define rational functions $r_{i}(t):=p_{i} / q$ for $i=0, \ldots, 6$. If $y_{0}, \ldots, y_{6}$ denote the (locally defined) solutions on the branches then for each $t$ we have that $y_{0}(t), \ldots, y_{6}(t)$ are the roots of $F(t, y)=0$ and it follows that

$$
y^{7}+r_{6}(t) y^{6}+\cdots+r_{1}(t) y+r_{0}(t)=\left(y-y_{0}(t)\right)\left(y-y_{1}(t)\right) \cdots\left(y-y_{6}(t)\right) .
$$

Thus, expanding the product on the right, the rational functions $r_{i}$ are obtained as symmetric polynomials in the $y_{i}$ :

$$
r_{0}=-y_{0} \cdots y_{6}, \ldots, r_{6}=-y_{0}-\cdots-y_{6} .
$$

Since the $r_{i}$ are global rational functions, the Puiseux expansions of the $y_{i}$ give the Laurent expansions at 0 of the $r_{i}$. Clearly only a finite number of terms of each Laurent expansion are required to determine each $r_{i}$, and indeed it is simple to convert these truncated Laurent expansions into global rational functions. Clearing the denominators then yields the solution curve, as in equation (7) of the introduction.

One may easily check on a computer that this curve has precisely the right monodromy over the $t$-line (and in particular is genus zero, and has monodromy group $A_{7}$ ). Also one finds that it has 10 singular points; 6 double points over $\mathbb{C} \backslash\{0,1\}$ and 4 more serious singularities over the branch points. Finally since it is a genus zero curve we can look for a rational parameterisation. Using the CASA package [17, and a simple Mobius transformation, we find the solution may be parameterised quite simply as:

$$
y=-\frac{\left(5 s^{2}-8 s+5\right)\left(7 s^{2}-7 s+4\right)}{s(s-2)(s+1)(2 s-1)\left(4 s^{2}-7 s+7\right)}, \quad t=\frac{\left(7 s^{2}-7 s+4\right)^{2}}{s^{3}\left(4 s^{2}-7 s+7\right)^{2}} .
$$

Note that the polynomial $F$ defining the solution curve is quite canonical but there are many possible parameterisations. Using the parameterisation it is easy to carry out the ultimate test and substitute back into the Painlevé VI equation (with parameters $(\alpha, \beta, \gamma, \delta)=(9,-4,4,45) / 98)$ finding that we do indeed have a solution. 


\section{InEQUivalence Theorem}

We know (cf. Remark 14 above and [13] Remark 0.2) that the five 'platonic' solutions of 13 are equivalent (via Okamoto transformations) to solutions associated to finite subgroups of $\mathrm{SL}_{2}(\mathbb{C})$. In other words, even though the unipotent matrices (24) generate an infinite group, there is an equivalent solution with finite $2 \times 2$ monodromy group.

This raises the following question: Even though the $2 \times 2$ monodromy data (22) associated to the Klein solution generates an infinite group, is there an equivalent solution with finite $2 \times 2$ monodromy? We will prove this is not the case:

Theorem 5. Suppose there is an algebraic solution of some Painlevé VI equation which is equivalent to the Klein solution under Okamoto's affine $F_{4}$ action. Then the corresponding $2 \times 2$ monodromy data $\left(M_{1}, M_{2}, M_{3}\right)$ also generate an infinite subgroup of $\mathrm{SL}_{2}(\mathbb{C})$.

Proof. First the parameters $\left(\theta_{1}, \theta_{2}, \theta_{3}, \theta_{4}\right)$ should be equivalent to the corresponding parameters $(2,2,2,4) / 7$ of the Klein solution. If $\left(M_{1}, M_{2}, M_{3}\right)$ generate a binary tetrahedral, octahedral or icosahedral group then we will not be able to get any sevens in the denominators (since these groups have no elements of order seven) so any solution associated to these groups is inequivalent to the Klein solution. (This uses the simple observation that Okamoto's transformations act within the ring $\mathbb{Z}\left[\frac{1}{2}, \theta_{1}, \theta_{2}, \theta_{3}, \theta_{4}\right]$ so that if the $\theta_{i}$ are rational numbers with no sevens in the denominators, then no equivalent set of parameters has a seven in any denominator.)

Next, suppose $F(t, y)=0$ is the curve defining the Klein solution and $F_{1}\left(t_{1}, y_{1}\right)=0$ is the curve defining the equivalent solution. Then we know ([30] p.361) that $t, t_{1}$ are related by a Mobius transformation permuting $0,1, \infty$.

Lemma 27. There is an isomorphism of the curves $F(t, y)=0$ and $F_{1}\left(y_{1}, t_{1}\right)=0$ covering the automorphism of the projective line mapping $t$ to $t_{1}$.

Proof. Let us recall some facts about Okamoto's transformations from [30, 29, 28]. First write $q:=y, q_{1}:=y_{1}$. Then, from the formulae for the action of the Okamoto transformations [29] Table 1, 28] (7.14), we see that $q_{1}$ is a rational function of $q, p, t$, where $p$ is the conjugate variable to $q$ in the Hamiltonian formulation of Painlevé VI (cf. [30] (0.6)). The first of Hamilton's equations says $\frac{d q}{d t}=\frac{\partial H}{\partial p}$, where $H=H_{V I}$ is the Hamiltonian [30] p.348. By observing that $H$ is a quadratic polynomial in $p$ (and rational in $t$ and polynomial in $q$ ) we deduce immediately that $p$ is a rational function of $\frac{d q}{d t}, q$ and $t$. Moreover since $q=y$ satisfies the polynomial equation $F(t, y)=0$ implicit differentiation enables us to express $\frac{d q}{d t}$ as a rational function of $q, t$. Thus $p$ is a rational function of just $q, t$ and so in turn $q_{1}$ is a rational function of just $q, t$.

Now by the symmetry of the situation the same argument also shows $q$ is a rational function of $q_{1}, t_{1}$. This sets up an isomorphism between the fields $\mathbb{C}(q, t) \cong \mathbb{C}\left(q_{1}, t_{1}\right)$ extending the isomorphism $\mathbb{C}(t) \cong \mathbb{C}\left(t_{1}\right)$ given by mapping $t$ to $t_{1}$. Dualising this gives the desired isomorphism of the corresponding curves.

In particular we see that $F_{1}$ must have degree seven in $y_{1}$, since the curves have the same number of branches. This implies $\left(M_{1}, M_{2}, M_{3}\right)$ cannot generate a cyclic group, since cyclic groups are abelian and so the pure braid group acts trivially; all such solutions have just one branch. 
Finally we need to rule out the binary dihedral groups which will need more work. Write the elements of the binary dihedral group of order $4 d$ as

$$
\widetilde{I_{2}(d)}=\left\{1, \zeta, \ldots, \zeta^{2 d-1}, \tau, \tau \zeta, \ldots, \tau \zeta^{2 d-1}\right\}
$$

where $\zeta:=\left(\begin{array}{cc}\varepsilon & \varepsilon^{-1}\end{array}\right), \tau:=\left(\begin{array}{cc}0 & -1 \\ 1 & 0\end{array}\right)$ and $\varepsilon=\exp (\pi i / d)$.

Below we will abbreviate $\tau \zeta^{a}$ as just $\tau a$ and $\zeta^{a}$ as $a$.

The basic strategy is to go through all possible triples of elements and show in each case that, on conjugacy classes, the generators $p_{1}:=\beta_{1}^{2}, p_{2}:=\beta_{2}^{2}$ of the pure braid group action cannot have two two-cycles and a three-cycle.

The basic relations we will use repeatedly are:

$$
\tau \zeta^{k}=\zeta^{-k} \tau, \quad \zeta^{2 d}=1
$$

First let us record the formulae for the action of $p_{1}$ on all possible pairs of elements. (Here $\beta_{1}$ acts by mapping a pair $(x, y)$ of elements to $\left(y, y^{-1} x y\right)$, and $p_{1}$ is the square of $\beta_{1}$.)

Lemma 28. Suppose $a, b$ are arbitrary integers. Then, in abbreviated form:

$$
\begin{gathered}
p_{1}(a, b)=(a, b), \\
p_{1}(\tau a, b)=(\tau(a+2 b),-b), \\
p_{1}(a, \tau b)=(-a, \tau(b-2 a)), \\
p_{1}(\tau a, \tau b)=(\tau(2 b-a), \tau(3 b-2 a)) .
\end{gathered}
$$

Proof. Straightforward computation.

Now, on triples, $\beta_{1}$ (respectively $\left.\beta_{2}\right)$ maps $(x, y, z)$ to $\left(y, y^{-1} x y, z\right)\left(\operatorname{resp} .\left(x, z, z^{-1} y z\right)\right)$. Immediately we see that any triple of the form

$$
(a, b, c), \quad(\tau a, b, c), \quad \text { or } \quad(a, b, \tau c)
$$

will be fixed by one or both of $p_{1}, p_{2}$. Thus the corresponding permutation representation will have a one-cycle, which is not permitted.

In general the triples of elements of $\widetilde{I_{2}(d)}$ fall into eight 'types' depending on if each element contains a $\tau$ or not. From Lemma $28 p_{1}$ and $p_{2}$ clearly take triples to triples of the same type. After the three types already dealt with the next four are:

$$
(a, \tau b, c), \quad(\tau a, \tau b, c), \quad(a, \tau b, \tau c), \quad(\tau a, b, \tau c) .
$$

For each of these one finds, from Lemma 28, that either $p_{1}^{2}$ or $p_{2}^{2}$ (or both) act trivially. This implies that there will be no three-cycles in the permutation representation of one or both of $p_{1}$ or $p_{2}$ on conjugacy classes of such triples.

Finally we need to rule out the triples of type $(\tau a, \tau b, \tau c)$. First let us note that the conjugacy class of $\tau$ has size $d$ and contains the elements $\tau(2 a)$ for any integer $a$, and the conjugacy class of $\tau \zeta$ has size $d$ and contains the elements $\tau(2 a+1)$. It follows that, upto overall conjugacy, we have:

$$
\begin{gathered}
p_{1}(\tau a, \tau b, \tau c) \cong(\tau a, \tau b, \tau(c-k)) \quad \text { where } k:=2(b-a), \text { and } \\
p_{2}(\tau a, \tau b, \tau c) \cong(\tau(a-l), \tau b, \tau c) \quad \text { where } l:=2(c-b) .
\end{gathered}
$$

Moreover the only (possibly distinct) triple of the form $(\tau p, \tau b, \tau q)$ that is conjugate to $(\tau a, \tau b, \tau c)$ is $(\tau(2 b-a), \tau b, \tau(2 b-c))$, which is obtained by conjugating by $\tau b$. 
Lemma 29. Let $o(k)$ be the order of the element $\zeta^{k}$ where $k=2(b-a)$. Then in the permutation representaion of $p_{1}$ the conjugacy classes of the triple through $(\tau a, \tau b, \tau c)$ lies in a cycle of length $o(k)$.

Proof. First if $\tau a=\tau(2 b-a)$ then $\zeta^{k}=1$ so $o(k)=1$ and (41) says the conjugacy class of $(\tau a, \tau b, \tau c)$ is fixed by $p_{1}$.

Secondly if $\tau a \neq \tau(2 b-a)$, i.e. $o(k)>1$ then by (41) we see $p_{1}^{r}(\tau a, \tau b, \tau c) \cong$ $(\tau a, \tau b, \tau(c-r k))$. This is conjugate to $(\tau a, \tau b, \tau c)$ if and only if $\zeta^{-r k}=1$ (using the fact that $\tau(2 b-a) \neq \tau a)$ i.e. if and only if $r$ is divisible by $o(k)$. Thus we are in a cycle of length $o(k)$.

Similarly if $o(l)$ is the order of $\zeta^{l}$ where $l=2(c-b)$ then the conjugacy class of $(\tau a, \tau b, \tau c)$ is in a cycle of $p_{2}$ of length $o(l)$.

Thus in order to be equivalent to the Klein solution we need $o(k), o(l) \in\{2,3\}$ for all the possible $k$ 's and $l$ 's that occur in the orbit. It is straightforward to check this is not possible: First from (41) note that $p_{1}$ maps the pair of integers $[k, l]$ to $[k, l-2 k]$ and similarly $p_{2}[k, l]=[k+2 l, l]$. Thus:

i) If $o(k)=o(l)=2$ then $o(l-2 k)=o(k+2 l)=2$ and, repeating, we see only two-cycles appear in the orbit, whereas we need a three-cycle.

ii) If $o(k)=2, o(l)=3$ then $o(k+2 l)=6$ and so we get an unwanted six-cycle. (Similarly if $o(k)=3, o(l)=2$.)

iii) If $o(k)=o(l)=3$ then $\zeta^{k+2 l}, \zeta^{l-2 k}$ each have order either one or three. Thus either an unwanted one-cycle appears or we only get three cycles; no two-cycles appear.

Thus we conclude that the Klein solution is not equivalent to any solution coming from a finite subgroup of $\mathrm{SL}_{2}(\mathbb{C})$.

\section{ReCOnstruCtion}

Given a triple $r_{1}, r_{2}, r_{3}$ of generators of a three-dimensional complex reflection group, we have explained how to obtain an $\mathrm{SL}_{2}\left(\mathbb{C}\right.$ ) triple $M_{1}, M_{2}, M_{3}$ (in an isomorphic braid group orbit) and then how, if Jimbo's formula is applicable, to obtain an algebraic solution $y(t)$ to the sixth Painlevé equation.

In this section we will explain how to obtain from $y(t)$ a rank three Fuchsian system with four poles on $\mathbb{P}^{1}$ and monodromy conjugate to the original complex reflection group (generated by three reflections).

First we recall (from [24]) that the solution $y(t)$ and its derivative determine algebraically an $\mathfrak{s l}_{2}$ system

$$
\frac{d \Phi}{d z}=A(z) \Phi ; \quad A(z)=\sum_{i=1}^{3} \frac{A_{i}}{z-a_{i}}
$$

with monodromy $\left(M_{1}, M_{2}, M_{3}\right)$, where $\left(a_{1}, a_{2}, a_{3}\right)=(0, t, 1)$, with respect to some choice of loops generating the fundamental group of the four-punctured sphere. (The exact formulae will be given below.) Now define

$$
\widehat{A}_{i}=A_{i}+\theta_{i} / 2
$$


for $i=1,2,3$, so that $\widehat{A}_{i}$ has rank one (and eigenvalues $\left\{0, \theta_{i}\right\}$ ). Then the system

$$
\frac{d}{d z}-\sum_{i=1}^{3} \frac{\widehat{A}_{i}}{z-a_{i}}
$$

has monodromy $\left(\widehat{M}_{1}, \widehat{M}_{2}, \widehat{M}_{3}\right)$ where $\widehat{M}_{i}=M_{i} \exp \left(\pi \sqrt{-1} \theta_{i}\right)$, which are pseudo-reflections in $\mathrm{GL}_{2}(\mathbb{C})$. Write these rank one matrices as

$$
\widehat{A}_{i}=h_{i} \otimes \gamma_{i} \quad \text { for some } h_{i} \in \mathbb{C}^{2}, \gamma_{i} \in\left(\mathbb{C}^{2}\right)^{*}, i=1,2,3 .
$$

In general the span of the $h_{i}$ will be two dimensional and without loss of generality we will suppose that $h_{1}, h_{2}$ are linearly independent (otherwise we can relabel below). Now consider the three $3 \times 3$ rank one matrices given by

$$
B_{i}:=\left(\begin{array}{cc}
0 & c_{i} \gamma_{i} \\
0 & \widehat{A}_{i} \\
0 &
\end{array}\right) \quad i=1,2,3
$$

for some constants $c_{1}, c_{2}, c_{3} \in \mathbb{C}$ and the corresponding rank three system

$$
\frac{d}{d z}-\sum_{i=1}^{3} \frac{B_{i}}{z-a_{i}}
$$

By overall conjugation, since $h_{1}, h_{2}$ are linearly independent, we can always assume $c_{1}=$ $c_{2}=0$. Now if $c_{3}=0$ then (44) is block diagonal and reduces to (43). However if $c_{3} \neq 0$ we obtain a rank three system with

$$
B_{i}=f_{i} \otimes \beta_{i}
$$

for a basis $f_{i}$ of $V:=\mathbb{C}^{3}$-namely:

$$
f_{1}=\left(\begin{array}{c}
0 \\
h_{1}
\end{array}\right), f_{2}=\left(\begin{array}{c}
0 \\
h_{2}
\end{array}\right), f_{3}=\left(\begin{array}{c}
c_{3} \\
h_{3}
\end{array}\right), \quad \beta_{i}=\left(\begin{array}{ll}
0 & \gamma_{i}
\end{array}\right) .
$$

Moreover, up to overall conjugation this system is independent of the choice of nonzero $c_{3}$ (since conjugating by $\operatorname{diag}(c, 1,1)$ scales $c_{3}$ arbitrarily).

In particular the invariant functions of the monodromy of the system (44) are independent of $c_{3}$ and are equal to the invariants of the monodromy of the limiting system with $c_{3}=0$, since the invariants are holomorphic functions of any parameters.

Now we can perform the scalar shift of section [3] in reverse. Namely, in the $f_{i}$ basis $\left(B_{1}, B_{2}, B_{3}\right)$ have the form

$$
B_{1}=\left(\begin{array}{ccc}
\widetilde{\lambda}_{1} & b_{12} & b_{13} \\
0 & 0 & 0 \\
0 & 0 & 0
\end{array}\right), \quad B_{2}=\left(\begin{array}{ccc}
0 & 0 & 0 \\
b_{21} & \widetilde{\lambda}_{3} & b_{23} \\
0 & 0 & 0
\end{array}\right), \quad B_{3}=\left(\begin{array}{ccc}
0 & 0 & 0 \\
0 & 0 & 0 \\
b_{31} & b_{32} & \widetilde{\lambda}_{3}
\end{array}\right)
$$

for some numbers $b_{i j}, \widetilde{\lambda}_{i}$. Then the scalar shift just translates each $\widetilde{\lambda}_{i}$ by the same scalar. If (as we are assuming) we started with a solution $y(t)$ as constructed with the procedure of this paper then $\widetilde{\lambda}_{i}=\lambda_{i}-\mu_{1}$, where $\lambda_{i}, \mu_{i}$ are related as in (19) to the original complex reflections $r_{1}, r_{2}, r_{3}$. 
Theorem 6. The system obtained by replacing each $\widetilde{\lambda}_{i}$ by $\lambda_{i}$ in (45) has monodromy conjugate to $\left(r_{1}, r_{2}, r_{3}\right)$. In other words there is a choice of fundamental solution $\Phi$ and of simple positive loops $l_{i}$ around $a_{i}$ for $i=1,2,3$ generating $\pi_{1}\left(\mathbb{P}^{1} \backslash\left\{a_{1}, a_{2}, a_{3}, \infty\right\}\right)$ such that $\Phi$ has monodromy $r_{i}$ around $l_{i}$.

Proof. Consider the system obtained by replacing $\widetilde{\lambda}_{i}$ by $\lambda_{i}+\lambda$ for each $i$, for varying $\lambda$ (so $\lambda=-\mu_{1}$ is the original system). Write

$$
\widehat{\mathbf{t}}(\lambda)=\left(t_{i}^{2}(\lambda), t_{i j}(\lambda), t_{321}(\lambda), t_{321}^{\prime}(\lambda)\right)
$$

for the invariant functions of the monodromy of the corresponding system. These functions vary holomorphically with $\lambda$ for any $\lambda \in \mathbb{C}$.

Write $r_{i}^{\prime}=1+e_{i} \otimes \alpha_{i}, u_{i j}=\alpha_{i}\left(e_{j}\right)$ for the monodromy data at $\lambda=0$. By construction the eigenvalues of $r_{1}^{\prime}, r_{2}^{\prime}, r_{3}^{\prime}$ and of the product $r_{3}^{\prime} r_{2}^{\prime} r_{1}^{\prime}$ are the same as those of the $r_{i}$ (since they are determined by the residues of the Fuchsian system).

Now the invariants $\widehat{\mathbf{t}}(0)$ are easily expressed in terms of $u$ (cf. proof of Lemma 2) and we know how $u$ varies with $\lambda$ (Theorem 3). It follows that

$$
\widehat{\mathbf{t}}(\lambda)=\left(h^{2} t_{i}^{2}, h^{2} t_{i j}, h^{2} t_{321}, h^{4} t_{321}^{\prime}\right)
$$

where $\left(t_{i}^{2}, t_{i j}, t_{321}, t_{321}^{\prime}\right)=\widehat{\mathbf{t}}(0)$ and $h=\exp (\pi i \lambda)$.

By construction we know the invariants $\widehat{\mathbf{t}}\left(-\mu_{1}\right)$ of the original system, namely they equal the invariants of the block diagonal monodromy data

$$
\left(\begin{array}{cc}
1 & \\
& \widehat{M}_{1}
\end{array}\right), \quad\left(\begin{array}{cc}
1 & \\
& \widehat{M}_{2}
\end{array}\right), \quad\left(\begin{array}{cc}
1 & \\
& \widehat{M}_{3}
\end{array}\right),
$$

which is the monodromy of the limiting system with $c_{3}=0$.

But this was set up precisely so that $\widehat{\mathbf{t}}(0)$ (obtained by inverting (46) when $\lambda=-\mu_{1}$ ) are the invariants of the original complex reflection group generators.

Finally we remark that the conjugacy class of $\left(r_{1}, r_{2}, r_{3}\right)$ is uniquely determined by the value of the invariants $\widehat{\mathbf{t}}$. This will be clear in the example below and follows in general from the fact that the invariants $\widehat{\mathbf{t}}$ generate the ring of conjugation invariant functions on triples of pseudo-reflections, and that the triple $\left(r_{1}, r_{2}, r_{3}\right)$ is irreducible.

Remark 30. Having established that the resulting system has the correct monodromy, let us record a more direct way to go from the $\widehat{A}_{i}$ to the $B_{i}$ of (45). The key point is that the pairwise, and three-fold, traces of distinct $B_{i}$ 's are independent of the scalar shift $\lambda$ (and that the constant $c_{3}$ does not contribute). Thus if $i \neq j$ then we find

$$
\begin{gathered}
b_{i j} b_{j i}=\operatorname{Tr}\left(B_{i} B_{j}\right)=\operatorname{Tr}\left(\widehat{A}_{i} \widehat{A}_{j}\right), \\
b_{32} b_{21} b_{13}=\operatorname{Tr}\left(B_{3} B_{2} B_{1}\right)=\operatorname{Tr}\left(\widehat{A}_{3} \widehat{A}_{2} \widehat{A}_{1}\right) .
\end{gathered}
$$

In general these are sufficient to determine $\left(B_{1}, B_{2}, B_{3}\right)$ uniquely up to conjugacy.

Now we will recall (from [24]) the formulae for the $\widehat{A}_{i}$ in terms of $y, y^{\prime}$. Let us first go in the other direction, and then invert. Consider the following rank one matrices

$$
\widehat{A}_{1}:=\left(\begin{array}{cc}
z_{1}+\theta_{1} & -u z_{1} \\
\left(z_{1}+\theta_{1}\right) / u & -z_{1}
\end{array}\right), \widehat{A}_{2}:=\left(\begin{array}{cc}
z_{2}+\theta_{2} & -w z_{2} \\
\left(z_{2}+\theta_{2}\right) / w & -z_{2}
\end{array}\right), \widehat{A}_{3}:=\left(\begin{array}{cc}
z_{3}+\theta_{3} & -v z_{3} \\
\left(z_{3}+\theta_{3}\right) / v & -z_{3}
\end{array}\right)
$$


so that $\widehat{A}_{i}$ has eigenvalues $\left\{0, \theta_{i}\right\}$ for $i=1,2,3$. Now if we define

$$
k_{1}:=\left(\theta_{4}-\theta_{1}-\theta_{2}-\theta_{3}\right) / 2, \quad k_{2}:=\left(-\theta_{4}-\theta_{1}-\theta_{2}-\theta_{3}\right) / 2
$$

and impose the equations

$$
z_{1}+z_{2}+z_{3}=k_{2}, \quad u z_{1}+v z_{3}+w z_{2}=0, \quad\left(z_{1}+\theta_{1}\right) / u+\left(z_{3}+\theta_{3}\right) / v+\left(z_{2}+\theta_{2}\right) / w=0
$$

then $\widehat{A}_{1}+\widehat{A}_{2}+\widehat{A}_{3}=-\operatorname{diag}\left(k_{1}, k_{2}\right)$, and the corresponding $\mathfrak{s l}_{2}$ matrices satisfy $A_{1}+A_{2}+$ $A_{3}=-\operatorname{diag}\left(\theta_{4},-\theta_{4}\right) / 2$.

Now we wish to define two $T$-invariant functions $x, y$ on the set of such triples $\left(\widehat{A}_{1}, \widehat{A}_{2}, \widehat{A}_{3}\right)$, where the one-dimensional torus $T \subset \mathrm{SL}_{2}(\mathbb{C})$ acts by diagonal conjugation. (The function $x$ is denoted $\widetilde{z}$ in $[24$.) First note that the $(1,2)$ matrix entry of

$$
\widehat{A}:=\frac{\widehat{A}_{1}}{z}+\frac{\widehat{A}_{2}}{z-t}+\frac{\widehat{A}_{3}}{z-1}
$$

is of the form $\frac{p(z)}{z(z-1)(z-t)}$ for some linear polynomial $p(z)$. Thus $\widehat{A}_{12}$ has a unique zero on the complex plane and we define $y$ to be the position of this zero. Explicitly one finds:

$$
y=\frac{t u z_{1}}{(t+1) u z_{1}+t v z_{3}+w z_{2}} .
$$

Then we define

$$
x=\frac{z_{1}}{y}+\frac{z_{2}}{y-t}+\frac{z_{1}}{y-1}
$$

which is clearly $T$-invariant. Note that if we set $z=y$ then $\widehat{A}$ is lower triangular and its first eigenvalue (i.e. its top-left entry) is $x+\frac{\theta_{1}}{y}+\frac{\theta_{2}}{y-t}+\frac{\theta_{3}}{y-1}$.

Now the fact is that we can go backwards and express the six variables $\left\{z_{1}, z_{2}, z_{3}, u, v, w\right\}$ in terms of $x, y$. That is, given $x, y$ we wish to solve the five equations (47), (48), (49) in the six unknowns $\left\{z_{1}, z_{2}, z_{3}, u, v, w\right\}$. To fix up the expected one degree of freedom we impose a sixth equation

$$
(t+1) u z_{1}+t v z_{3}+w z_{2}=1
$$

so that (48) now says $y=t u z_{1}$. (This degree of freedom corresponds to the torus action mentioned above.) One then finds, algebraically, that these six equations in the six unknowns admit the unique solution:

$$
\begin{gathered}
z_{1}=y \frac{E-k_{2}^{2}(t+1)}{t \theta_{4}}, \quad z_{2}=(y-t) \frac{E+t \theta_{4}(y-1) x k_{2}^{2}-t k_{1} k_{2}}{t(t-1) \theta_{4}} \\
z_{3}=-(y-1) \frac{E+\theta_{4}(y-t) x-k_{2}^{2} t-k_{1} k_{2}}{(t-1) \theta_{4}}, \\
u=\frac{y}{t z_{1}}, \quad v=-\frac{y-1}{(t-1) z_{3}}, \quad w=\frac{y-t}{t(t-1) z_{2}}
\end{gathered}
$$

where

$E=y(y-1)(y-t) x^{2}+\left(\theta_{3}(y-t)+t \theta_{2}(y-1)-2 k_{2}(y-1)(y-t)\right) x+k_{2}^{2} y-k_{2}\left(\theta_{3}+t \theta_{2}\right)$.

Finally we recall that if $y(t)$ solves PVI then the variable $x$ is directly expressible in terms of the derivative of $y$ (cf. [24] above C55): 


$$
x=\frac{1}{2}\left(\frac{t(t-1) y^{\prime}}{y(y-1)(y-t)}-\frac{\theta_{1}}{y}-\frac{\theta_{3}}{y-1}-\frac{\theta_{2}+1}{y-t}\right) .
$$

Thus given a solution $y(t)$ to PVI we may use these formulae to reconstruct the matrices $\widehat{A}_{1}, \widehat{A}_{2}, \widehat{A}_{3}$ upto overall conjugation by the diagonal torus.

Although it will not be needed here we remark that one needs to do a further quadrature in order for the $\widehat{A}_{i}$ to solve Schlesinger's equations; they need to vary appropriately within the torus orbit. This is done via the variable $k$ of [24], which evolves according to the linear differential equation 24] C55.

Example. For the Klein solution, suppose we set the parameter $s=5 / 4$ (this is chosen to give reasonably simple numbers below). Then $t=121 / 125$ and the above formulae yield (cf. Remark 30):

$$
\begin{gathered}
b_{12} b_{21}=\frac{3}{224}, \quad b_{23} b_{32}=\frac{249}{2464}, \quad b_{13} b_{31}=\frac{5}{176}, \\
b_{32} b_{21} b_{13}=\frac{21}{1408} .
\end{gathered}
$$

These values determine $\left(B_{1}, B_{2}, B_{3}\right)$ uniquely upto conjugacy, and it is easy to find a representative triple:

Corollary 31. Let

$$
B_{1}=\left(\begin{array}{ccc}
\frac{1}{2} & \frac{3}{224} & \frac{21}{1408} \\
0 & 0 & 0 \\
0 & 0 & 0
\end{array}\right), \quad B_{2}=\left(\begin{array}{ccc}
0 & 0 & 0 \\
1 & \frac{1}{2} & \frac{5}{176} \\
0 & 0 & 0
\end{array}\right), \quad B_{3}=\left(\begin{array}{ccc}
0 & 0 & 0 \\
0 & 0 & 0 \\
\frac{332}{49} & 1 & \frac{1}{2}
\end{array}\right) .
$$

Then the Fuchsian system

$$
\frac{d}{d z}-\left(\frac{B_{1}}{z}+\frac{B_{2}}{z-\frac{121}{125}}+\frac{B_{3}}{z-1}\right)
$$

has monodromy equal to the Klein complex reflection group, generated by reflections.

Remark 32. The author is grateful to M. van Hoeij and J.-A. Weil for confirming on a computer that this system does indeed admit an invariant of degree four.

\section{The $3 \times 3$ FuChSian REPRESENTATION OF PVI}

In this section we will describe the direct path to the sixth Painlevé equation from the $3 \times 3$ isomonodromic deformations we have been considering. Then we will explain how to deduce a recent theorem of Inaba-Iwasaki-Saito [21] from the results of this paper.

Let $V=\mathbb{C}^{3}$ and suppose $B_{1}, B_{2}, B_{3} \in \operatorname{End}(V)$ have rank one, linearly independent images and $\operatorname{Tr}\left(B_{i}\right)=\lambda_{i}$. Suppose that $B_{1}+B_{2}+B_{3}$ is diagonalisable with eigenvalues $\mu_{1}, \mu_{2}, \mu_{3}$ and write

$$
\nabla:=d-B d z, \quad B(z):=\frac{B_{1}}{z}+\frac{B_{2}}{z-t}+\frac{B_{3}}{z-1} .
$$

In section 3 we showed how isomonodromic deformations of $\nabla$ lead to $\mathfrak{s l}_{2}$ isomonodromic deformations which are well-known to be equivalent to PVI. (Note we are setting the pole positions $a_{i}$ of section 3 to be $\left(a_{1}, a_{2}, a_{3}\right)=(0, t, 1)$.) One may go directly to PVI as follows (this was stated without proof in [6] Remark 4). First conjugate $B_{1}, B_{2}, B_{3}$ 
by a single element of $\mathrm{GL}_{3}(\mathbb{C})$ such that $B_{1}+B_{2}+B_{3}=\operatorname{diag}\left(\mu_{1}, \mu_{2}, \mu_{3}\right)$. Consider the polynomial $p(z)$ defined to be the 2,3 matrix entry of

$$
z(z-1)(z-t) B(z) .
$$

By construction $p(z)$ is a linear polynomial, so has a unique zero on the complex plane. Define $y$ to be the position of this zero.

Proposition 33. If we vary t and evolve B according to Schlesinger's equations (26) then $y(t)$ solves the PVI equation with parameters determined by $\left\{\lambda_{i}, \mu_{j}\right\}$ as in (18), (20).

Proof. As in section 3 we perform the scalar shift by $\lambda=-\mu_{1}$. Under this shift $B \mapsto B^{\prime}$ say. Then $B_{1}^{\prime}+B_{2}^{\prime}+B_{3}^{\prime}=\operatorname{diag}\left(0, \mu_{2}-\mu_{1}, \mu_{3}-\mu_{1}\right)$ and we deduce $B_{i}^{\prime}\left(e_{1}\right)=0$ in this basis, for each $i$. Then the bottom-right $2 \times 2$ submatrix $\widehat{A}$ of $B^{\prime}$ also solves the Schlesinger equations and the standard theory [24] says the position of the zero of the top-right entry of $\widehat{A}$ solves a PVI equation. The parameters of this PVI equation are as in (18), (20). Clearly the position of the zero of the 1,2 entry of $\widehat{A}$ is the position of the zero of the 2,3 entry of $B^{\prime}$. It remains to check this equals that of $B$, i.e. before the scalar shift. This is not obvious, but may be seen as follows.

Suppose we have performed the scalar shift by arbitrary $\lambda$. Write $c_{i}(\lambda):=\left(B_{i}^{\prime}\right)_{23}$. We claim $c_{i} / c_{j}$ is independent of $\lambda$ for all $i, j$. This easily implies the position of the zero of $p(z)$ is independent of $\lambda$ as required. To deduce the claim write $B_{i}=f_{i} \otimes \beta_{i}$ for a basis $\left\{f_{i}\right\}$. Let $\left\{\widehat{f}_{i}\right\}$ be the dual basis. Write $\Delta:=\operatorname{diag}\left(\mu_{1}, \mu_{2}, \mu_{3}\right)$. Since $B_{1}+B_{2}+B_{3}=\Delta$ we find $\beta_{i}:=\widehat{f}_{i} \circ B_{i}=\widehat{f_{i}} \circ \Delta$ so that $B_{i}=f_{i} \otimes \widehat{f_{i}} \Delta$. Thus upon shifting we find $B_{i}^{\prime}=f_{i} \otimes \widehat{f_{i}}(\Delta+\lambda)$. Hence $c_{i}=\widehat{e}_{2} B_{i}^{\prime} e_{3}=\left(f_{i} \otimes \widehat{f}_{i}\right)_{23} \times\left(\mu_{3}+\lambda\right)$ and $c_{i} / c_{j}=\left(f_{i} \otimes \widehat{f}_{i}\right)_{23} /\left(f_{j} \otimes \widehat{f}_{j}\right)_{23}$ which is manifestly independent of $\lambda$.

Of course the 2,3 matrix entry of $B$ is not particularly special. One can conjugate $B$ by a permutation matrix to move any of the other off-diagonal entries of $B$ into that position. We will show these solve equivalent Painlevé VI equations, related by Okamoto transformations. (Note that such conjugation corresponds to permuting the $\mu_{i}$.)

Let $S_{3}$ denote the symmetric group on three letters, which is generated by the transpositions $\alpha=(12), \beta=(13)$. On one hand $S_{3}$ acts on $B$ via permutation matrices, permuting $\mu_{1}, \mu_{2}, \mu_{3}$ arbitrarily. On the other hand we may map $S_{3}$ into the group of Okamoto transformations by sending

$$
\alpha \mapsto s_{1} s_{2} s_{1}, \quad \beta \mapsto\left(s_{0} s_{1} s_{3} s_{4}\right) s_{2}\left(s_{0} s_{1} s_{3} s_{4}\right) .
$$

Here each $s_{i}$, from [29], is a generator of Okamoto's affine $D_{4}$ symmetry group of PVI, which acts on the set of PVI equations taking solutions to solutions.

Lemma 34. Suppose $\sigma \in S_{3}$ and let $P$ be the corresponding permutation matrix and let $s$ be the corresponding element of affine $D_{4}$. If we vary $t$ and evolve $B$ according to Schlesinger's equations (26) then the PVI solution $y(t)$ associated to $P B P^{-1}$ is the transform, via the transformation $s$, of that associated to $B$.

Proof. Let $y_{0}$ be the original solution from Proposition 33. We wish to show $y=s\left(y_{0}\right)$. From $B$ and $P B P^{-1}$ we get two $2 \times 2$ systems $\widehat{A}, \widehat{A}^{\prime}$ respectively. It is straightforward to check their parameters are related by $s$ (cf. Lemma 9). Thus $y, s\left(y_{0}\right)$ solve the same PVI 
equation. Now from Remark 30] we deduce that for any distinct $i, j, k$

$$
\operatorname{Tr}\left(\widehat{A}_{i} \widehat{A}_{j}\right)=\operatorname{Tr}\left(\widehat{A}_{i}^{\prime} \widehat{A}_{j}^{\prime}\right) \quad \text { and } \quad \operatorname{Tr}\left(\widehat{A}_{i} \widehat{A}_{j} \widehat{A}_{k}\right)=\operatorname{Tr}\left(\widehat{A}_{i}^{\prime} \widehat{A}_{j}^{\prime} \widehat{A}_{k}^{\prime}\right),
$$

since the $P$ 's cancel in the traces. Now we point out the algebraic fact that if two solutions are related by $s$ then (51) holds for the corresponding $2 \times 2$ systems (and generically the converse is true: (51) and that the parameters match implies the solutions match).

Corollary 35 (Inaba-Iwasaki-Saito [21]). Let $s$ be any element of Okamoto's affine $D_{4}$ symmetry group (not necessarily in the $S_{3}$ subgroup considered above). By definition $s$ acts birationally on the space of $2 \times 2$ systems $A$. This action preserves the quadratic functions $m_{12}, m_{23}, m_{13}$ of the monodromy matrices of these systems.

Proof. The affine $D_{4}$ action has five generators $s_{i}$ for $i=0,1,2,3,4$. (We use the notation of [29; the indices are permuted in [21].) The result is simple for $s_{0}, s_{1}, s_{3}, s_{4}$, since each of these transformations arise as a type of gauge transformation and fixes all the monodromy data (cf. [21] Section 6-that these cases are trivial is stated [21] p.15).

The hard part is to establish the result for $s_{2}$. However we have shown that $s_{1} s_{2} s_{1}$ is the transformation which comes from swapping $\mu_{1}$ and $\mu_{2}$. On the level of $3 \times 3$ monodromy data this corresponds to just swapping $n_{1}, n_{2}$ (recall $\left.n_{j}=\exp \left(\pi i \mu_{j}\right)\right)$. However glancing at the map $\varphi$ of (17) we recall that $m_{i j}=t_{i j} / t_{i} t_{j}$, which does not involve either $n_{1}, n_{2}$ and so is fixed.

Remark 36. One reason we are interested in this result here is to check that up to equivalence there is just one Klein solution. Recall [6] that there are exactly two braid group orbits of conjugacy classes of triples of generating reflections of the Klein complex reflection group, containing the triples

$$
\left(r_{1}, r_{2}, r_{3}\right) \quad \text { and } \quad\left(r_{3}, r_{2}, r_{1}\right)
$$

respectively. It is easy to see they have PVI parameters which are equivalent under the affine $D_{4}$ group (and have isomorphic $\mathcal{P}_{3}$ orbits). But one would like to check the actual PVI solutions are equivalent. This is facilitated by Corollary 35; it is sufficient to check the quadratic functions of the corresponding $2 \times 2$ monodromy data match up. In turn, via $\varphi$, this amounts to checking that $\operatorname{Tr}\left(r_{1} r_{2}\right)=\operatorname{Tr}\left(r_{3} r_{2}\right)$. But we saw in section 2 both sides equal 1.

\section{Appendix A.}

We wish to explain how to extract Theorem 3] from the paper [1] of Balser, Jurkat and Lutz.

Let us briefly recall the set-up of [1. Given an $n \times n$ matrix $A_{1}$ and a diagonal matrix $B_{0}=\operatorname{diag}\left(b_{1}, \ldots, b_{n}\right)$ (with the $b_{i}$ pairwise distinct) one considers the Fuchsian connection ([1] $(0.2))$

$$
d-\left(B_{0}-z\right)^{-1}\left(1+A_{1}\right) d z=d-\sum_{i=1}^{n} \frac{-E_{i}\left(1+A_{1}\right)}{z-b_{i}} d z
$$


where $E_{i}$ is the $n \times n$ matrix with a one in its $i, i$ entry and is otherwise zero. (To avoid confusion with other notation of the present paper we have relabelled $t \mapsto z, \Lambda \mapsto B_{0}, \lambda_{i} \mapsto$ $b_{i}$ from [1.) Write $\Lambda^{\prime}=\operatorname{diag}\left(\lambda_{1}^{\prime}, \ldots, \lambda_{n}^{\prime}\right)$ for the diagonal part of $A_{1}$ and suppose that

i) No $\lambda_{i}^{\prime}$ is an integer, and that

ii) No eigenvalue of $A_{1}$ is an integer.

Condition $i$ ) implies that each residue of (52) is rank one and has non-integral trace.

Now one chooses an admissible branch cut direction $\eta$ and cuts the complex $z$-plane from each $b_{i}$ to $\infty$ along the direction $\eta$, leaving a simply connected domain $\mathcal{P}_{\eta} \subset \mathbb{C}$. (In fact ([1] p.694) one takes $\eta \in \mathbb{R}$ and uses $\eta$ to give logarithm choices on $\mathcal{P}_{\eta}$ near each $b_{i}$.) The direction $\eta$ is said to be 'admissible' if none of these cuts overlap, and the inadmissible $\eta$ in the interval $(-\pi / 2,3 \pi / 2]$ are labelled $\eta_{0}, \ldots, \eta_{m-1}$ (with $\left.\eta_{i+1}<\eta_{i}\right)$. This labelling is extended to all integral subscripts $\nu$ by setting $\eta_{\nu}=\eta_{\nu+k m}+2 \pi k$ for any integer $k$.

Now, given an admissible $\eta$ one may canonically construct a certain fundamental solution $Y^{*}(z)$ of (52) on $\mathcal{P}_{\eta}$ and define an $n \times n$ matrix $C=C(\eta)$, with ones on the diagonal, such that (by [1] Lemma 1): After continuing $Y^{*}$ along a small positive loop around $b_{k}$ (and crossing the $k$ th cut), $Y^{*}$ becomes

$$
Y^{*}\left(1+C_{k}^{*}\right)
$$

where $C_{k}^{*}$ is zero except for its $k$ th column which equals the $k$ th column of $C \widetilde{D}$, where $\widetilde{D}:=\exp \left(-2 \pi i \Lambda^{\prime}\right)-1$.

If $\eta$ varies through admissible values then $C(\eta)$ does not change. Thus we choose an integer $\nu$ and let $C=C_{\nu}$ be $C(\eta)$ for any $\eta \in\left(\eta_{\nu+1}, \eta_{\nu}\right)$, as in [1] Remark 3.1 p.699.

Thus if we suppose (1] p.697) that, when looking along $\eta$ towards infinity, that $b_{k+1}$ lies to the right of $b_{k}$ for $k=1, \ldots, n-1$, then the monodromy of $Y^{*}$ around a large positive loop encircling all the $b_{i}$ is the product of pseudo-reflections

$$
\left(1+C_{1}^{*}\right) \cdots\left(1+C_{n}^{*}\right)
$$

The main facts we need from [1] now are:

1) That the Stokes matrices $C_{\nu}^{ \pm}$of the irregular connection

$$
d-\left(B_{0}+\frac{A_{1}}{x}\right) d x
$$

are determined by $C_{\nu}$ by the equation

$$
C_{\nu} D=C_{\nu}^{+}-e^{2 \pi i \Lambda^{\prime}} C_{\nu}^{-}
$$

where $D:=1-\exp 2 \pi i \Lambda^{\prime}$. (This is equation (3.25) of [1], and that the $C_{\nu}^{ \pm}$are the Stokes matrices is the content of [1] Theorem 2, p.714.)

2) That the Stokes matrices are unchanged if $A_{1}$ undergoes a scalar shift $A_{1} \mapsto A_{1}-\lambda$ (1] Remark 4.4, p.712).

This is sufficient to determine how the pseudo-reflections $1+C_{k}^{*}$ vary under the scalar shift; one doesn't need to know how the Stokes matrices are defined, only that they are triangular matrices with ones on the diagonal. The main subtlety one needs to appreciate is that: in the above convention (with $b_{k+1}$ to the right of $b_{k}$ ) then

$$
C_{\nu}^{+} \text {is lower triangular and } C_{\nu}^{-} \text {is upper triangular. }
$$

Indeed ([1] p.701, paragraph before (3.16)) $C_{\nu}^{+/-}$are upper/lower triangular respectively if $b_{1}, \ldots, b_{n}$ are ordered according to the dominance relation on $S_{\nu+1}^{\prime}$. This dominance 
relation is defined (top of p.699) so that it coincides with the natural ordering of the indices if $b_{1}, \ldots, b_{n}$ are ordered so that the $j$ th cut (along the direction $\eta \in\left(\eta_{\nu+1}, \eta_{\nu}\right)$ ) lies to the right of the $k$ th cut whenever $j<k$ (again looking along $\eta$ towards infinity). This is opposite to the previous ordering of the $b_{i}$. Thus sticking to our original ordering we deduce (54).

In summary if we set $V=\mathbb{C}^{n}$ and write $1+C_{k}^{*}=1+v_{k} \otimes \beta_{k}$ (where $\left\{\beta_{i}\right\}$ is the standard basis of $V^{*}$ and $v_{k} \in V$ is the $k$ th column of $v:=C_{\nu} \widetilde{D}$, so $\left.v_{i j}=\beta_{i}\left(v_{j}\right)\right)$ then

$$
v=C_{\nu} \widetilde{D}=\left(C_{\nu}^{+}-e^{2 \pi i \Lambda^{\prime}} C_{\nu}^{-}\right) D^{-1} \widetilde{D} \sim e^{-2 \pi i \Lambda^{\prime}} C_{\nu}^{+}-C_{\nu}^{-}
$$

where we note that $e^{2 \pi i \Lambda^{\prime}} \widetilde{D}=D$ and where $\sim$ is defined so that $A \sim B$ if there is an invertible diagonal matrix $s$ such that $A=s B s^{-1}$. (This conjugation by $s$ just corresponds to different choices of $v_{k}, \beta_{k}$ such that $1+C_{k}^{*}=1+v_{k} \otimes \beta_{k}$ and so clearly does not affect the corresponding pseudo-reflections.)

Thus under the scalar shift, the upper triangular part of $v$ is fixed, the lower triangular part is scaled by $\exp (2 \pi i \lambda)$ and the diagonal part $e^{-2 \pi i \Lambda^{\prime}}-1$ is changed to $e^{-2 \pi i\left(\Lambda^{\prime}-\lambda\right)}-1$.

Finally let us relate this back to our conventions in the body of the paper. Namely we have a connection

$$
d-\sum \frac{B_{i}}{z-a_{i}} d z
$$

with rank one residues, monodromy $r_{i}$ around $a_{i}$ and monodromy $r_{n} \cdots r_{1}$ around a large positive loop. The images of the $B_{i}$ make up a basis of $V$ so we may conjugate $\left(B_{1}, \ldots, B_{n}\right)$ such that each $B_{i}$ is zero except in row $(n-i+1)$.

Then we set $b_{i}=a_{n-i+1}$ and define $A_{1}=-1-\sum B_{i}$ so that

$$
\frac{-E_{i}\left(1+A_{1}\right)}{z-b_{i}}=\frac{B_{n-i+1}}{z-a_{n-i+1}}
$$

for each $i$ and that

$$
\left(1+C_{1}^{*}, \ldots, 1+C_{n}^{*}\right)=\left(r_{n}, \ldots, r_{1}\right)
$$

upto overall conjugation. Thus if we write $r_{i}=1+e_{i} \otimes \alpha_{i}$ and define $u$ by $u_{i j}=\alpha_{i}\left(e_{j}\right)$ we have that

$$
u \sim \Omega v \Omega
$$

where $\Omega$ is the order reversing permutation matrix $\left(\Omega_{i j}=\delta_{i n-j+1}\right)$. Note that we denoted the trace of $B_{i}$ as $\lambda_{i}$ so that the diagonal part $\Lambda^{\prime}$ of $A_{1}$ is

$$
\Lambda^{\prime}=-1-\Omega \Lambda \Omega
$$

where $\Lambda:=\operatorname{diag}\left(\lambda_{1}, \ldots, \lambda_{n}\right)$. Thus, if we write

$$
u=t^{2} u_{+}-u_{-}
$$

with $t^{2}$ diagonal and $u_{+/-}$upper/lower triangular with ones on the diagonal, we have that, upto overall conjugation by a diagonal matrix:

$$
u_{+}=\Omega C_{\nu}^{+} \Omega, \quad u_{-}=\Omega C_{\nu}^{-} \Omega
$$

and $t^{2}=\exp (2 \pi i \Lambda)$. Therefore under the scalar shift both $u_{ \pm}$are fixed and so $u$ is changed to $h^{2} t^{2} u_{+}-u_{-}$, establishing Theorem 3 . 
Remark 37. One can check independently that it is the upper triangular part of $u$ that should be scaled by $h^{2}$, rather than the lower triangular part, since we know that the eigenvalues of $r_{n} \cdots r_{1}$ should be scaled by $h^{2}$. Indeed if we expand

$$
\begin{gathered}
\operatorname{Tr}\left(r_{n} \cdots r_{1}\right)=n+\sum_{i} u_{i i}+\sum_{i>j} u_{i j} u_{j i}+\sum_{i>j>k} u_{i j} u_{j k} u_{k i}+\cdots+u_{n n-1} u_{n-1 n-2} \cdots u_{21} u_{1 n} \\
=\sum_{i} t_{i}+\sum_{i>j} u_{i j} u_{j i}+\sum_{i>j>k} u_{i j} u_{j k} u_{k i}+\cdots+u_{n n-1} u_{n-1 n-2} \cdots u_{21} u_{1 n}
\end{gathered}
$$

we see that scaling just the upper triangular part of $u$ (and the $t_{i}$ ) scales each term here by $h^{2}$ as required, and otherwise one obtains higher powers of $h$.

\section{Appendix B.}

We will explain how to derive the formula for the parameter $s$ in Jimbo's formula (40). This formula is stated incorrectly, and not derived, in [23. Since it is not immediately clear how to derive the formula we sketch the main steps here, and point out the (probably typographical) error. (We remark that the whole procedure described in the present paper does not work without this correction.)

Suppose we have four matrices $M_{j} \in \mathrm{SL}_{2}(\mathbb{C}), j=0, t, 1, \infty$ satisfying

$$
M_{\infty} M_{1} M_{t} M_{0}=1 \text {, }
$$

and $M_{j}$ has eigenvalues $\left\{\exp \left( \pm \pi i \theta_{j}\right)\right\}$ where $\theta_{j} \notin \mathbb{Z}$. Write $\varepsilon_{\infty}=\exp \left(\pi i \theta_{\infty}\right)$ and suppose $M_{\infty}$ is actually diagonal $\left(M_{\infty}=\operatorname{diag}\left(\varepsilon_{\infty}, \varepsilon_{\infty}^{-1}\right)\right)$. Define $\sigma_{j k} \in \mathbb{C}$ with $0 \leq \operatorname{Re}\left(\sigma_{j k}\right) \leq 1$ (for $j, k \in\{0, t, 1, \infty\}$ ) by

$$
\operatorname{Tr}\left(M_{j} M_{k}\right)=2 \cos \left(\pi \sigma_{j k}\right),
$$

and let $\sigma:=\sigma_{0 t}$ and $\varepsilon:=\exp (\pi i \sigma)$.

Under the further assumptions that $\sigma$ is nonzero, that $0 \leq \operatorname{Re}(\sigma)<1$ and that none of the eight numbers

$$
\theta_{0} \pm \theta_{t} \pm \sigma, \quad \theta_{0} \pm \theta_{t} \mp \sigma, \quad \theta_{\infty} \pm \theta_{1} \pm \sigma, \quad \theta_{\infty} \pm \theta_{1} \mp \sigma
$$

is an even integer, Jimbo [23] p.1141 points out that, up to overall conjugacy by a diagonal matrix, $M_{0}, M_{t}, M_{1}$ are given, for some $s \in \mathbb{C}^{*}$, by:

$$
\begin{gathered}
\left(i s_{\sigma}\right) M_{0}=C^{-1}\left(\begin{array}{cc}
\varepsilon c_{0}-c_{t} & 2 s \alpha^{\prime} \gamma^{\prime} \\
-2 s^{-1} \beta^{\prime} \delta^{\prime} & -\varepsilon^{-1} c_{0}+c_{t}
\end{array}\right) C, \quad\left(i s_{\sigma}\right) M_{t}=C^{-1}\left(\begin{array}{cc}
\varepsilon c_{t}-c_{0} & -2 s \varepsilon \alpha^{\prime} \gamma^{\prime} \\
2 s^{-1} \varepsilon^{-1} \beta^{\prime} \delta^{\prime} & -\varepsilon^{-1} c_{t}+c_{0}
\end{array}\right) C \\
\left(i s_{\infty}\right) M_{1}=\left(\begin{array}{cc}
c_{\sigma}-\varepsilon_{\infty}^{-1} c_{1} & -2 \varepsilon_{\infty}^{-1} \beta \gamma \\
2 \varepsilon_{\infty} \alpha \delta & -c_{\sigma}+\varepsilon_{\infty} c_{1}
\end{array}\right), \quad \text { where } \quad C=\left(\begin{array}{cc}
\delta & \beta \\
\alpha & \gamma
\end{array}\right)
\end{gathered}
$$

and we have used the temporary notation:

$$
\begin{gathered}
c_{j}:=\cos \left(\pi \theta_{j}\right), \quad c_{\sigma}:=\cos (\pi \sigma), \quad s_{j}:=\sin \left(\pi \theta_{j}\right), \quad s_{\sigma}:=\sin (\pi \sigma), \\
\alpha=\sin \frac{\pi}{2}\left(\theta_{\infty}-\theta_{1}+\sigma\right), \beta=\sin \frac{\pi}{2}\left(\theta_{\infty}+\theta_{1}+\sigma\right), \gamma=\sin \frac{\pi}{2}\left(\theta_{\infty}+\theta_{1}-\sigma\right), \delta=\sin \frac{\pi}{2}\left(\theta_{\infty}-\theta_{1}-\sigma\right), \\
\alpha^{\prime}=\sin \frac{\pi}{2}\left(\theta_{0}-\theta_{t}+\sigma\right), \beta^{\prime}=\sin \frac{\pi}{2}\left(\theta_{0}+\theta_{t}+\sigma\right), \gamma^{\prime}=\sin \frac{\pi}{2}\left(\theta_{0}+\theta_{t}-\sigma\right), \delta^{\prime}=\sin \frac{\pi}{2}\left(\theta_{0}-\theta_{t}-\sigma\right) .
\end{gathered}
$$

Notice that $\sigma_{01}$ and $\sigma_{1 t}$ do not appear in these formulae; The idea now is to express the parameter $s$ in terms of $\sigma_{01}, \sigma_{1 t}$ (and the other parameters). Naively we can just calculate $\operatorname{Tr}\left(M_{0} M_{1}\right)$ and $\operatorname{Tr}\left(M_{1} M_{t}\right)$ from the above formulae, equate them with 
$2 \cos \left(\pi \sigma_{01}\right), 2 \cos \left(\pi \sigma_{1 t}\right)$ respectively and try to solve for $s$. However this yields a complicated expression in the trigonometric functions and a simple formula looks beyond reach.

The key observation to simplify the computation is that the above parameterisation of the matrices is such that $C M_{t} M_{0} C^{-1}$ is diagonal and equal to $\Delta:=\operatorname{diag}\left(\varepsilon, \varepsilon^{-1}\right.$ ) (and also equal to $C M_{1}^{-1} M_{\infty}^{-1} C^{-1}$ by (55) ). Thus we find

$$
\begin{aligned}
& 2 \cos \left(\pi \sigma_{01}\right)=\operatorname{Tr}\left(M_{0} M_{1}\right)=\operatorname{Tr}\left(C M_{\infty}^{-1} C^{-1} \Delta^{-1}\left(C M_{0} C^{-1}\right)\right) \\
& 2 \cos \left(\pi \sigma_{1 t}\right)=\operatorname{Tr}\left(M_{1} M_{t}\right)=\operatorname{Tr}\left(C M_{\infty}^{-1} C^{-1} \Delta^{-1}\left(C M_{t} C^{-1}\right)\right)
\end{aligned}
$$

whose right-hand sides are more manageable expressions in the trigonometric functions, and are linear in $1, s, s^{-1}$. If we take the combination (56) $+\varepsilon(157)$ then the $s^{-1}$ terms cancel and upon rearranging we find:

$$
\begin{aligned}
2 i \operatorname{det}(C) s_{\sigma}\left(c_{01}+\varepsilon c_{1 t}\right)= & \left(\gamma \delta \varepsilon_{\infty}^{-1}-\alpha \beta \varepsilon_{\infty}\right)\left(\varepsilon-\varepsilon^{-1}\right) c_{2}+ \\
& \left(\gamma \delta \varepsilon_{\infty}-\alpha \beta \varepsilon_{\infty}^{-1}\right)\left(\varepsilon^{2}-1\right) c_{0}+2 s \alpha \gamma \alpha^{\prime} \gamma^{\prime}\left(\varepsilon_{\infty}-\varepsilon_{\infty}^{-1}\right)\left(\varepsilon-\varepsilon^{-1}\right)
\end{aligned}
$$

where $c_{01}=\cos \left(\pi \sigma_{01}\right)$ and $c_{1 t}=\cos \left(\pi \sigma_{1 t}\right)$. To proceed we note:

\section{Lemma 38.}

a) $\gamma \delta-\alpha \beta:=\operatorname{det}(C)=-s_{\infty} s_{\sigma}$

b) $\gamma \delta \varepsilon_{\infty}^{-1}-\alpha \beta \varepsilon_{\infty}=i s_{\infty}\left(\varepsilon c_{\infty}-c_{1}\right)$

c) $\gamma \delta \varepsilon_{\infty}-\alpha \beta \varepsilon_{\infty}^{-1}=i s_{\infty}\left(c_{1}-\varepsilon^{-1} c_{\infty}\right)$

Proof. A few applications of standard trigonometric formulae yield

$$
\alpha \beta=\left(c_{1}-\left(c_{\infty} c_{\sigma}-s_{\infty} s_{\sigma}\right)\right) / 2, \quad \gamma \delta=\left(c_{1}-\left(c_{\infty} c_{\sigma}+s_{\infty} s_{\sigma}\right)\right) / 2
$$

which gives $a$ ) immediately and also enable $b$ ),c) to be easily deduced.

Substituting these into (58) and cancelling a factor of $2 s_{\sigma} s_{\infty}=-\left(\varepsilon-\varepsilon^{-1}\right)\left(\varepsilon_{\infty}-\varepsilon_{\infty}^{-1}\right) / 2$ yields the desired formula:

$$
s=\frac{\varepsilon\left(i s_{\sigma} c_{1 t}-c_{t} c_{\infty}-c_{0} c_{1}\right)+i s_{\sigma} c_{01}+c_{t} c_{1}+c_{\infty} c_{0}}{4 \alpha \gamma \alpha^{\prime} \gamma^{\prime}} .
$$

This differs from formula (1.8) of [23] in a single sign, in $\alpha$, which was crucial for us.

\section{REFERENCES}

1. W. Balser, W.B. Jurkat, and D.A. Lutz, On the reduction of connection problems for differential equations with an irregular singularity to ones with only regular singularities, I., SIAM J. Math. Anal. 12 (1981), no. 5, 691-721.

2. P. P. Boalch, The icosahedral solutions to the sixth Painleve equation, in preparation.

3. _ Stokes matrices, Poisson Lie groups and Frobenius manifolds, Invent. math. 146 (2001), $479-506$.

4. __ Symplectic manifolds and isomonodromic deformations, Adv. in Math. 163 (2001), 137-205.

5. _ G-bundles, isomonodromy and quantum Weyl groups, Int. Math. Res. Not. (2002), no. 22, 1129-1166, math.DG/0108152.

6. __ Painlevé equations and complex reflections, Ann. Inst. Fourier 53 (2003), no. 4, 1009-1022.

7. R. Carter, Simple groups of Lie type, Wiley, New York, 1972.

8. A. J. Coleman, Killing and Coxeter transformations of Kac-Moody algebras, Invent. Math. 95 (1989), $447-477$. 
9. H. S. M. Coxeter, The product of the generators of a finite group generated by reflections, Duke Math. J. 18 (1951), 765-782. MR 13,528d

10. C. De Concini, V. G. Kac, and C. Procesi, Quantum coadjoint action, J. Amer. Math. Soc. 5 (1992), no. $1,151-189$.

11. B. Dubrovin, Geometry of 2D topological field theories, Integrable Systems and Quantum Groups (M.Francaviglia and S.Greco, eds.), vol. 1620, Springer Lect. Notes Math., 1995, pp. 120-348.

12. _ Painlevé transcendents in two-dimensional topological field theory, The Painlevé property, Springer, New York, 1999, pp. 287-412, (math/9803107).

13. B. Dubrovin and M. Mazzocco, Monodromy of certain Painlevé-VI transcendents and reflection groups, Invent. Math. 141 (2000), no. 1, 55-147. MR 2001j:34114

14. R. Fricke and F. Klein, Vorlesungen über die Theorie der automorphen Funktionen. I, Druck und Verlag von B. G. Teubner, Leipzig, 1897, p. 366.

15. D. Guzzetti, The elliptic representation of the general Painlevé VI equation, Comm. Pure Appl. Math. 55 (2002), no. 10, 1280-1363. MR 2003f:34187

16. J. Harnad, Dual isomonodromic deformations and moment maps to loop algebras, Comm. Math. Phys. 166 (1994), 337-365.

17. R. Hemmecke, E. Hillgarter, and F. Winkler, The CASA system, Handbook of Computer Algebra: Foundation, Applications, Systems (J. Grabmeier, E. Kaltofen, and V. Weispfenning, eds.), Springer Verlag, Heidelberg, 2000, (see: www.risc.uni-linz.ac.at/software/casa).

18. N. J. Hitchin, Frobenius manifolds, Gauge Theory and Symplectic Geometry (J. Hurtubise and F. Lalonde, eds.), NATO ASI Series C: Maths \& Phys., vol. 488, Kluwer, 1995.

19. _ Poncelet polygons and the Painlevé equations, Geometry and analysis (Bombay, 1992), Tata Inst. Fund. Res., Bombay, 1995, pp. 151-185. MR 97d:32042

20. _ A lecture on the octahedron, Bull. London Math. Soc. 35 (2003), 577-600.

21. M. Inaba, K. Iwasaki, and M.-H. Saito, Bäcklund transformations of the sixth Painlevé equation in terms of Riemann-Hilbert correspondence, Int. Math. Res. Not. (2004), no. 1, 1-30, math.AG/0309341. MR 2036953

22. K. Iwasaki, A modular group action on cubic surfaces and the monodromy of the Painlevé VI equation, Proc. Japan Acad., Ser. A 78 (2002), 131-135.

23. M. Jimbo, Monodromy problem and the boundary condition for some Painlevé equations, Publ. Res. Inst. Math. Sci. 18 (1982), no. 3, 1137-1161. MR 85c:58050

24. M. Jimbo and T. Miwa, Monodromy preserving deformations of linear differential equations with rational coefficients II, Physica 2D (1981), 407-448.

25. M. Jimbo, T. Miwa, Y. Môri, and M. Sato, Density matrix of an impenetrable Bose gas and the fifth Painlevé transcendant, Physica 1D (1980), 80-158.

26. W. Killing, Die Zusammensetzung der stetigen endlichen Transformationsgruppen II, Math. Ann. 33 (1889), 1-48.

27. W. Magnus, Rings of Fricke characters and automorphism groups of free groups, Math. Z. (1980), 91-103.

28. T. Masuda, On a class of algebraic solutions to the Painlevé VI equation, its determinant formula and coalescence cascade, Funkcial. Ekvac. 46 (2003), no. 1, 121-171, nlin.SI/0202044. MR 2004e:34138

29. M. Noumi and Y. Yamada, A new Lax pair for the sixth Painlevé equation associated with $\hat{\mathfrak{s o}}(8)$, Microlocal Analysis and Complex Fourier Analysis (T. Kawai and K. Fujita, eds.), World Scientific, 2002, math-ph/0203029.

30. K. Okamoto, Studies on the Painlevé equations. I. Sixth Painlevé equation $P_{\mathrm{VI}}$, Ann. Mat. Pura Appl. (4) 146 (1987), 337-381. MR 88m:58062

31. G. C. Shephard and J. A. Todd, Finite unitary reflection groups, Canadian J. Math. 6 (1954), 274304. MR $15,600 \mathrm{~b}$

32. V. Toledano Laredo, A Kohno-Drinfeld theorem for quantum Weyl groups, Duke Math. J. 112 (2002), no. 3, 421-451. MR 1896470

École Normale Supérieure, 45 Rue d'Ulm, 75005 Paris, France

E-mail address: boalch@dma.ens.fr 